\title{
QUEEN'S
UNIVERSITY
BELFAST
}

\section{Photochemical Homologation for the Preparation of Aliphatic Aldehydes in Flow}

Chen, Y., Leonardi, M., Dingwall, P., Labes, R., Pasau, P., Blakemore, D. C., \& Ley, S. V. (2018).

Photochemical Homologation for the Preparation of Aliphatic Aldehydes in Flow. Journal of Organic Chemistry, 83(24), 15558-15568. https://doi.org/10.1021/acs.joc.8b02721

Published in:

Journal of Organic Chemistry

Document Version:

Publisher's PDF, also known as Version of record

Queen's University Belfast - Research Portal:

Link to publication record in Queen's University Belfast Research Portal

\section{Publisher rights}

Copyright 2018 the authors.

This is an open access article published under a Creative Commons Attribution License (https://creativecommons.org/licenses/by/4.0/), which permits unrestricted use, distribution and reproduction in any medium, provided the author and source are cited.

\section{General rights}

Copyright for the publications made accessible via the Queen's University Belfast Research Portal is retained by the author(s) and / or other copyright owners and it is a condition of accessing these publications that users recognise and abide by the legal requirements associated with these rights.

Take down policy

The Research Portal is Queen's institutional repository that provides access to Queen's research output. Every effort has been made to ensure that content in the Research Portal does not infringe any person's rights, or applicable UK laws. If you discover content in the Research Portal that you believe breaches copyright or violates any law, please contact openaccess@qub.ac.uk. 


\title{
Photochemical Homologation for the Preparation of Aliphatic Aldehydes in Flow
}

\author{
Yiding Chen, ${ }^{\dagger, \otimes}$ Marco Leonardi, ${ }^{\dagger, \downarrow, \perp, \otimes}$ Paul Dingwall, ${ }^{\dagger}$ Ricardo Labes, ${ }^{\dagger}$ (๑) Patrick Pasau, ${ }^{\S}$ \\ David C. Blakemore," and Steven V. Ley*,† \\ ${ }^{\dagger}$ Department of Chemistry, University of Cambridge, Lensfield Road, Cambridge CB2 1EW, U.K. \\ ${ }^{\ddagger}$ Departmento de Química en Ciencias Farmacéuticas, Facultad de Farmacia, Universidad Complutense, 28040 Madrid, Spain \\ ${ }^{\S}$ UCB Biopharma SPRL, Chemical Research R5, Chemin du Foriest, 1420 Braine-L'Alleud, Belgium \\ "Medicine Design, Pfizer, Inc., Eastern Point Road, Groton, Connecticut 06340, United States
}

\section{Supporting Information}

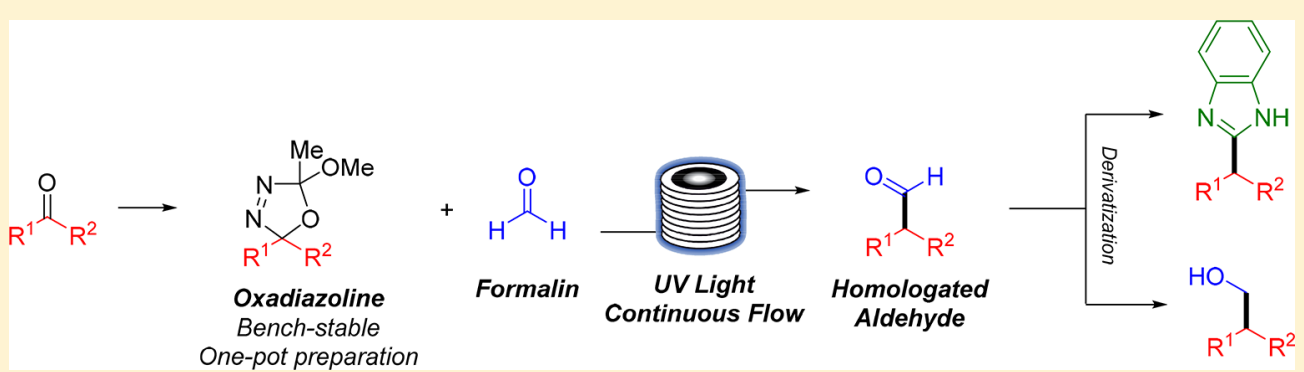

ABSTRACT: Cheap and readily available aqueous formaldehyde was used as a formylating reagent in a homologation reaction with nonstabilized diazo compounds, enabled by UV photolysis of bench-stable oxadiazolines in a flow photoreactor. Various aliphatic aldehydes were synthesized along with the corresponding derivatized alcohols and benzimidazoles. No transition-metal catalyst or additive was required to affect the reaction, which proceeded at room temperature in $80 \mathrm{~min}$.

$\mathrm{F}$ ollowing the discovery of the Buchner-Curtius-Schlotterbeck reaction over a century ago, ${ }^{1}$ the interactions between carbonyl compounds and diazo compounds have been extensively studied. ${ }^{2,3}$ These methods constitute a powerful synthetic tool for $\mathrm{C}-\mathrm{C}$ bond formation, especially for the extension of carbon chains and for the construction and decoration of ketones. ${ }^{4-6}$ However, the controlled formation of aldehyde products using diazo chemistry is not a simple task; carbonyl groups and diazo compounds are highly reactive coupling partners. The reliable and safe generation of nonstabilized diazo compounds is currently an area of intense research, ${ }^{7-10}$ and one our laboratory has been interested in due to the application of flow chemistry as an enabling technology ${ }^{11-13}$ to overcome the safety issues traditionally associated with diazo compounds. ${ }^{14-16}$ Following the pioneering work from Warkentin and co-workers, ${ }^{17,18}$ we have recently published two reports on the use of oxadiazolines as benchstable, nonstabilized diazo compound precursors and their application in protodeboronative and oxidative $\mathrm{C}\left(\mathrm{sp}^{2}\right)-\mathrm{C}\left(\mathrm{sp}^{3}\right)$ cross-coupling with boronic acids ${ }^{19}$ and aldehyde $\mathrm{C}-\mathrm{H}$ functionalization to afford unsymmetrical ketones. ${ }^{20}$

During this work, two reports in the literature caught our attention (Scheme 1). Kingsbury and co-workers demonstrated a Lewis acid catalyzed double homologation reaction by combining ex situ prepared diazo compounds and the flashpyrolyzed preparation of anhydrous formaldehyde (Scheme 1, A), ${ }^{21}$ and $\mathrm{Hu}$ et al. reported an interesting three-component
Scheme 1. Examples of homologation reactions involving diazo and carbonyl compounds

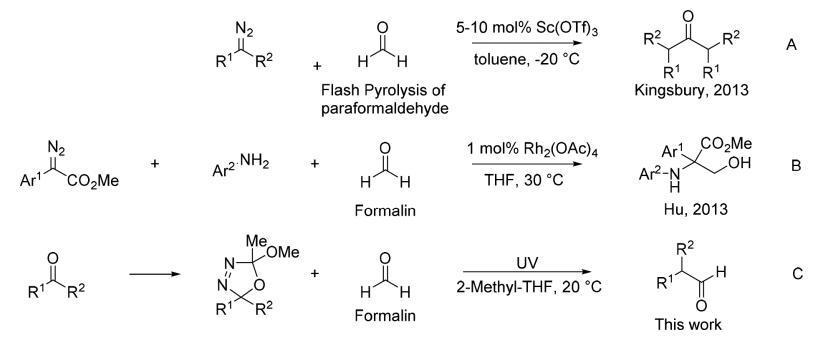

coupling of aryldiazoacetate, aniline, and aqueous formaldehyde (Scheme 1, B). ${ }^{22}$ Both of these reactions passed through, but did not stop at, the aldehyde oxidation state on the way to a final product, either the doubly homologated ketone or the $\alpha$-aryl serine derivative. These examples encouraged us to control the homologation reaction and stop at the aldehyde product in as simple a manner as possible and without the use of a protecting group strategy. Herein, we report the controlled homologation of nonstabilized diazo compounds generated from bench-stable precursors in flow to form aldehydes and their derivatives (Scheme 1, C).

Received: October 23, 2018

Published: November 16, 2018 
Our investigation began by combining 2-tetralone oxadiazoline 1a with different sources of formaldehyde under UV irradiation (Table 1). Common formaldehyde surrogates

\section{Table 1. Optimization of Aldehyde Formation}

\begin{tabular}{|c|c|c|c|c|c|c|}
\hline & ${ }_{2}{ }_{2}^{O}$ & ${ }_{V_{R}=10}^{9 w U V}$ & $\bigwedge_{\text {FlowiR }}$ & & $3 a$ & \\
\hline entry $^{a}$ & formaldehyde source & $\begin{array}{c}\text { ox } \\
(\mathrm{M})\end{array}$ & $\begin{array}{c}t_{\mathrm{R}} \\
(\mathrm{min})\end{array}$ & $\mathrm{T}\left({ }^{\circ} \mathrm{C}\right)$ & $\begin{array}{c}\text { conv } \\
(\%)\end{array}$ & $\begin{array}{c}\text { yield } \\
(\%)\end{array}$ \\
\hline 1 & dioxolane & 0.1 & 40 & 20 & 99 & 0 \\
\hline 2 & trioxane & 0.1 & 40 & 20 & 96 & 2 \\
\hline 3 & $\begin{array}{l}\text { thermolyzed } \\
\text { paraformaldehyde }\end{array}$ & 0.1 & 40 & 20 & 67 & 55 \\
\hline 4 & $37 \%$ aq & 0.1 & 40 & 20 & 78 & 48 \\
\hline 5 & $37 \%$ aq & 0.1 & 40 & 10 & 72 & 41 \\
\hline 6 & $37 \%$ aq & 0.05 & 40 & 10 & 80 & 39 \\
\hline 7 & $37 \%$ aq & 0.1 & 80 & 20 & 87 & ${ }^{60}(50)^{b}$ \\
\hline $8^{c}$ & $37 \%$ aq & 0.1 & 80 & 20 & 86 & 58 \\
\hline $9^{d}$ & $37 \%$ aq & 0.1 & 80 & 20 & 87 & 56 \\
\hline $10^{e}$ & $37 \%$ aq & 0.1 & 80 & 20 & 78 & 41 \\
\hline $11^{f}$ & $37 \%$ aq & 0.1 & 80 & 20 & 79 & 12 \\
\hline
\end{tabular}

${ }^{a}$ Reaction conditions: oxadiazoline $(0.4 \mathrm{mmol})$, formalin $(0.3 \mathrm{~mL}, 37$ wt $\%$, $4.0 \mathrm{mmol})$, 2-methyltetrahydrofuran $(4 \mathrm{~mL})$. NMR yields calculated with 1,3,5-trimethoxybenzene as an internal standard. ${ }^{b}$ Isolated yield. ${ }^{c} 100$ equiv of formaldehyde was used. ${ }^{d} 5.0$ equiv of formaldehyde was used. ${ }^{e}$ Tetrahydrofuran was used instead of 2methyltetrahydrofuran. ${ }^{f}$ Dichloromethane was used instead of 2methyltetrahydrofuran.

trioxane and dioxolane both delivered only trace amounts of the desired aldehyde product $3 \mathbf{a}$ despite almost complete conversion of the oxadiazoline starting material (entry 1 and $2) .^{23}$ While we established our first success using a stock solution of monomeric formaldehyde created via thermolysis of paraformaldehyde, ${ }^{24}$ resulting in a $55 \%$ yield of the desired aldehyde (entry 3 ), practical considerations of the procedure and the propensity of the stock solution to polymerize without warning on warming above $-78{ }^{\circ} \mathrm{C}$ made this an unattractive approach. We then turned our attention to formalin, a $37 \%$ aqueous solution of formaldehyde, which pleasingly gave a modest isolated yield (48\%) of the target aldehyde (3a, entry $4) .{ }^{25}$ Lowering the reaction temperature to $10{ }^{\circ} \mathrm{C}$ led to a diminished conversion and yield (entry 5), while a decrease in the reaction concentration did not result in an improved yield despite a higher conversion (entry 6). Elongating the residence time to $80 \mathrm{~min}$ improved both conversion (87\%) as well as yield $(60 \%)$ (entry 7 ). Formaldehyde ratio changes were ineffective (entry 8 and 9). Similarly, switching to tetrahydrofuran also marginally lowered the yield to $41 \%$, while with dichloromethane this dropped to $12 \%$ (entry 10 and 11).

After multiple reaction optimization attempts, we accepted the isolated yield of aldehyde of around 50\%, albeit with a higher conversion of the oxadiazoline. On further examination of the crude sample mixture, along with the required aliphatic aldehyde we found a significant amount of hydrated material was also present. At no time did we observe more than $10 \%$ of the doubly homologated ketone. We presume that formation of the hydrate, due to the presence of a large amount of water in the reaction media, acts as an in situ protecting group, and this, coupled with a very low concentration of diazo compound throughout the course of the reaction, disfavors double homologation. We further observed that over an extended period of time the corresponding carboxylic acid product was formed, most likely as a result of an aerobic oxidative transformation, which is not uncommon for aldehydes of this type.

Also owing to the volatility of some of the aldehydic products, we decided to directly reduce the crude mixture with sodium borohydride $\left(\mathrm{NaBH}_{4}\right)$, thereby converting the products into the corresponding alcohol (4a), resulting in an improved yield of $60 \%$ over two steps (Table 2 , entry 1 ). We also saw this procedure as a way of storing these unstable aliphatic aldehydes through recycling via a secondary oxidation process back to aldehydes should this be necessary. To further exemplify the method and to better capture the unstable and sometimes volatile small-molecule products, the crude aldehydes were additionally subjected to oxidative condensation with $o$-phenylenediamine following a modified procedure originally reported by Jiao et al. ${ }^{26}$ This procedure gave 2substituted benzimidazole (5a) from 1,2,3,4-tetrahydronaphthalene-2-carbaldehyde (3a) via in situ generated aliphatic aldehyde in an overall $72 \%$ isolated yield (Table 2, entry 1).

With these various conditions in hand, we set about examining the scope of the reactions (Table 2). Tetrahydropyran substrate (1b) was able to produce the corresponding aldehyde (3b) in a $48 \%$ yield while providing $53 \%$ of alcohol (4b) and $76 \%$ benzimidazole (5b). Similarly, tetrahydrothiopyran (1c), tetrahydrothiophene (1d), and cyclohexyldioxolo (1e) derivatives all underwent these three individual transformations to give products $(3 \mathbf{c}-\mathbf{e}, 4 \mathbf{c}-\mathbf{e}$, and $5 \mathbf{c}-\mathbf{e}$ ) in reasonable yields (entries 3,4 , and 5). As for nitrogen-based functional groups, Boc-protected amine (1f) and $N$-pyrimidinyl piperidine $(\mathbf{1 g})$ were also tolerated (entry 6 and 7 ). Bulky 2 -adamantyl aldehyde (3h) was isolated in $68 \%$ yield, together with $75 \%$ of 2 -adamantanemethanol $(4 \mathrm{~h})$ and $79 \%$ of 2 -adamantylbenzimidazole (5h). Lastly, cyclobutyl oxadiazoline (1i) did not give useful isolated yields owing to aldehyde and alcohol volatility ( $3 \mathbf{i}$ and $4 \mathbf{i}$ ), although the formation of 2-cyclobutylbenzimidazole was achieved in 59\% yield $(\mathbf{5 i})$.

Except for methoxynaphthalene substrate (3j, entry 10$)$, the $\alpha$-methyl aldehydes we obtained have displayed a tendency toward hydration or aerobic oxidation, thus resulting in low crude NMR yields and difficulty in isolation $(3 \mathbf{k}-\mathbf{p})$, which is well-known for similar materials. The efficiency of the reaction was generally better represented by comparing the yield of alcohols and benzimidazoles. In some cases, such as 5-hydroxy2-methylpentanal (3k), homologated product was identified as $81 \%$ of the hydrated form when only $4 \%$ of aldehyde was observed in NMR analysis, even though $66 \%$ of alcohol product (4k) was isolated over two steps. Pyridine (11) and furan (1m) were all successfully homologated into the corresponding products $(41, \mathrm{~m}, \mathbf{5 l}, \mathrm{m})$, respectively (entries 12 and 13). Alkyne- and alkene-substituted oxadiazolines (1n, 1o) both gave reasonable isolated yields as aldehyde derivatives (4n, 5n,o), with alkyne substrate produced lower yield arguably owing to larger steric hindrance (5o). Even though 2-cyclopropylpropanal (3p) and 2-cyclopropylpropan-1-ol $(4 p)$ were not able to give good isolated yields, the formation of $69 \%$ of 2-(1-cyclopropylethyl)benzimidazole ( $5 p$ ) proved the effectiveness of oxadiazoline as a successful diazo precursor for homologation. Many of these products can be thought of as branched, or iso, aldehydes which would be difficult to prepare 
Table 2. Scope and Derivatization of Oxadiazolines and Aqueous Formaldehyde

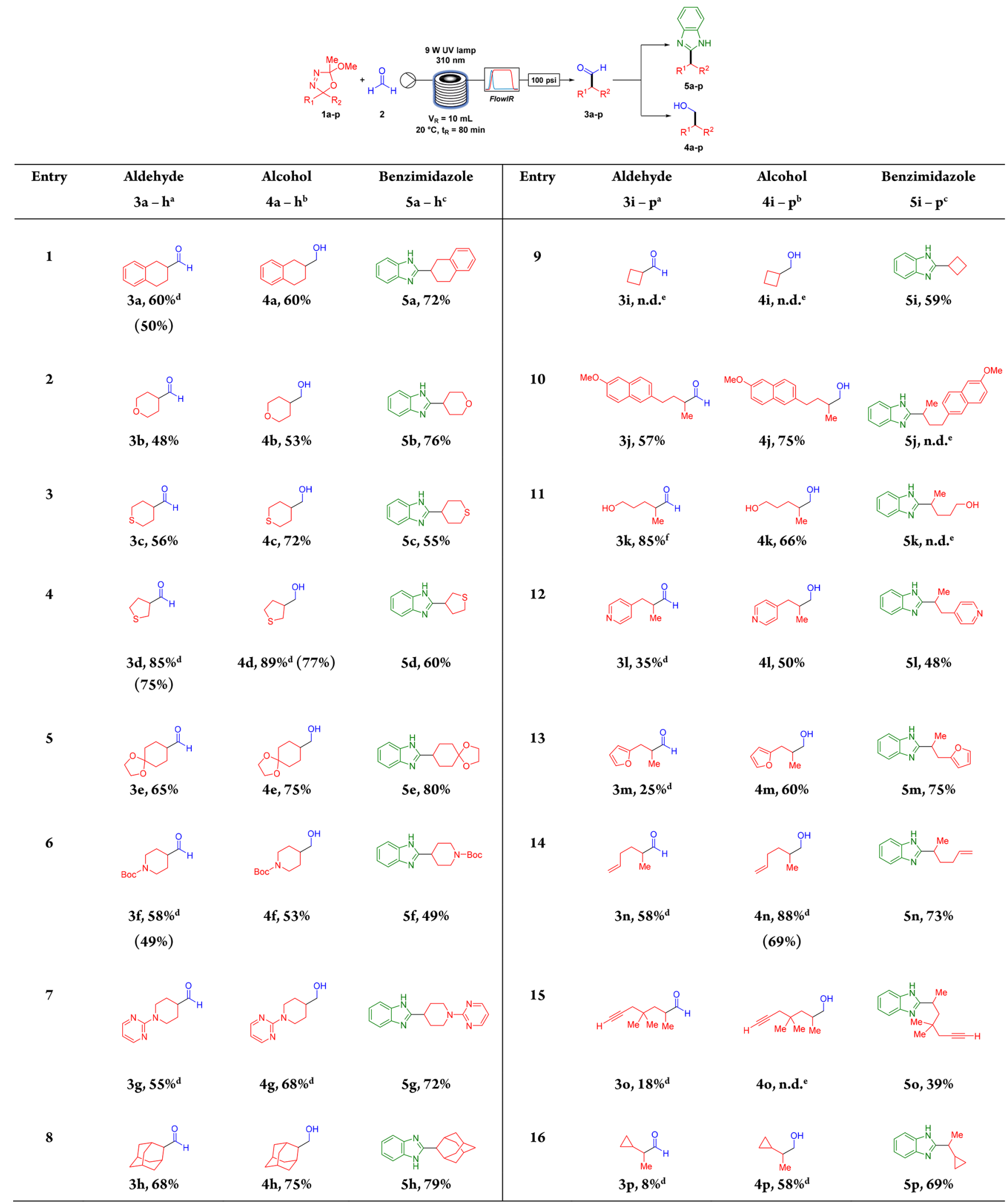

${ }^{a}$ Reaction conditions: oxadiazoline $\left(1.0\right.$ equiv, $0.1 \mathrm{M}$ ), formaldehyde (10 equiv, $37 \mathrm{wt} \%$ in $\left.\mathrm{H}_{2} \mathrm{O}, 1.0 \mathrm{M}\right)$ in 2 -methyltetrahydrofuran. ${ }^{b}$ Aldehyde reduced directly with $\mathrm{NaBH}_{4}(10$ equiv, $0.5 \mathrm{M})$ in ethanol. ${ }^{c}$ Aldehyde reacted with $o$-phenylenediamine $(1.5$ equiv, $0.075 \mathrm{M})$ in toluene. ${ }^{d} \mathrm{NMR}$ yield, calculated using 1,3,5-trimethoxybenzene as an internal standard. ${ }^{e}$ Not determined due to volatility or product contamination. $f_{81 \%}$ of the product identified as the hydrated form. 
through traditional methods such as hydroformylation, particularly in the presence of alkenes or alkynes.

The homologation reaction of oxadiazolines obtained from ketones has provided us with satisfying results toward $\alpha, \alpha$ disubstituted branched aliphatic aldehydes. However, similar oxadiazolines generated from aldehydes are difficult to obtain, which therefore obstructed the access toward linear aldehydes. To overcome this difficulty, we applied an alternative route to diazo compounds generated from hydrazones, prepared from the corresponding benzaldehydes according to our previously reported procedure. ${ }^{27,28}$ With the help of a glass static mixer chip, an ethyl acetate solution of diazo compound was combined with 37 wt \% aqueous formaldehyde solution in line, and the resulting homologated aldehyde product was collected in the output stream and purified (Table $3,7 \mathbf{a}-\mathbf{c}$ ) or

Table 3. Homologation of Aldehydes with Aqueous Formaldehyde via Hydrazine

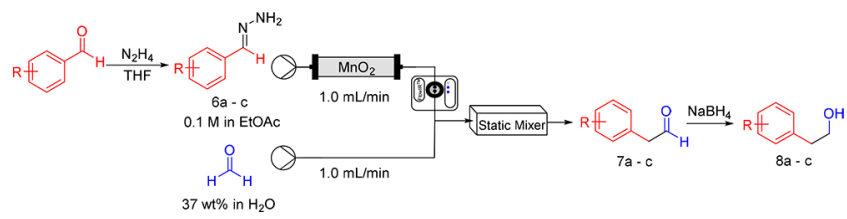

Entry Aldehyde $-7 \mathbf{a}-\mathbf{c}$

${ }^{a}$ Aldehyde extracted with ethyl acetate then reduced directly with $\mathrm{NaBH}_{4}$ (10 equiv, $0.5 \mathrm{M}$ ) in ethanol.

extracted and reduced directly to the corresponding alcohol in good yields $(\mathbf{8 a}-\mathbf{c})$. No attempt was made to further exemplify this procedure, although it should be noted that the method does overcome classical issues associated with phenacetaldehyde preparation.

We present a mild, operationally straightforward procedure for the overall homologation of ketones and aryl aldehydes via nonstabilized diazo compounds in flow. The route complements other homologation methods while avoiding expensive and reactive transition-metal catalysts and uses formalin as a cheap and readily available source of carbon.

\section{EXPERIMENTAL SECTION}

General Information. All batch reactions were performed under an atmosphere of nitrogen using oven-dried glassware unless otherwise stated. UV flow reactions were performed using a Vaportec E-series and UV-150 system. Hydrazone flow reactions were performed using a Uniqsis FlowSyn platform. Reagents were purchased from Sigma-Aldrich, Alfa Aesar, Acros, and Fluorochem and were used as supplied unless stated otherwise. 2-Methyltetrahydrofuran (2-MeTHF, anhydrous, inhibitor free, $\geq 99.9 \%$ ) and tetrahydrofuran (THF, anhydrous, inhibitor free, $\geq 99.9 \%$ ) were purchased from Sigma-Aldrich and used as supplied. Workup solvents were employed directly from commercial sources, i.e., Sigma-Aldrich, unless stated otherwise. Petroleum ether refers to the fractions of petroleum ether collected between 40 and $60{ }^{\circ} \mathrm{C}$ b.p.

Flash column chromatography was performed using a Biotage SPX system with single-use disposable silica columns of the appropriate size (SiliaSep Flash Cartridges, 4 or $12 \mathrm{~g}$ of $40-60 \mu \mathrm{m}$ ISO04/012). Analytical thin-layer chromatography (TLC) was performed using silica gel $60 \mathrm{~F} 254$ precoated glass-backed plates and visualized by ultraviolet radiation $(254 \mathrm{~nm}$ ) and appropriate dip (typically potassium permanganate or ninhydrin).

${ }^{1} \mathrm{H}$ NMR and ${ }^{13} \mathrm{C}\left\{{ }^{1} \mathrm{H}\right\}$ NMR spectra were recorded on a $600 \mathrm{MHz}$ Bruker DRX-600 spectrometer. Chemical shifts $(\delta)$ are referenced to the residual solvent as $\mathrm{CDCl}_{3}$ or DMSO- $d_{6}$ in parts per million (ppm). Signals are reported with the descriptions of their environments (e.g., ArH, NH, OH). Coupling constants $J$ are quoted in hertz $(\mathrm{Hz})$. Proton and carbon multiplicity is recorded as singlet (s), doublet $(\mathrm{d})$, triplet $(\mathrm{t})$, quartet $(\mathrm{q})$, multiplet $(\mathrm{m})$, and broad $(\mathrm{br})$ or a combinations thereof. All compounds examined were dried in vacuo to remove residual solvents. Spectra are assigned as fully as possible using ${ }^{1} \mathrm{H}$-tCOSY, DEPT-135, HSQC, and ${ }^{1} \mathrm{H}$ NOESY where appropriate to facilitate structural determination. Multiple signals arising from (pseudo)axial/equatorial positions are suffixed, for example, $H_{\mathrm{a}}$ and $H_{\mathrm{a}}{ }^{\prime} \cdot{ }^{1} \mathrm{H}$ NMR signals are reported to two decimal places and ${ }^{13} \mathrm{C}$ signals to one decimal place.

Infrared spectra were recorded neat on a PerkinElmer Spectrum One FTIR spectrometer with a universal ATR sampling accessory; selected peaks are reported.

Low-resolution mass spectrometry was performed on a Advion Expression CMS spectrometer. High-resolution mass spectrometry (HRMS) was performed using positive or negative electrospray ionization (ESI+) by the Mass Spectrometry Service for the Chemistry Department at the University of Cambridge.

Melting points were recorded on a Stanford Research Systems OptiMelt automated melting point system.

The oxadiazolines $\mathbf{1} \mathbf{a}-\mathbf{p}$ were synthesized according to the precedent literature procedure without further modifications. ${ }^{19}$ The hydrazones $6 \mathbf{a}-\mathbf{c}$ were synthesized according to the precedent procedure published by our group. ${ }^{28}$

All compounds listed in the paper are $>95 \%$ purity. Some products appear to be very hydroscopic and, therefore, contain $0.2-0.5$ molar equiv of water $\left(2-5\right.$ wt \%) in the ${ }^{1} \mathrm{H}$ NMR spectra as shown below. Volatile compounds are reported with minor solvents. Inseparable impurities are noted.

Synthesis of Aliphatic Aldehydes. General Procedure A for the Synthesis of Aliphatic Aldehydes. A solution of the appropriate oxadiazoline ( 1.0 equiv, $0.05 \mathrm{mmol} / \mathrm{mL}$ ) and formaldehyde (10 equiv of aqueous solution, $37 \% \mathrm{w} / \mathrm{w})$ in 2 -MeTHF $(0.5 \mathrm{~mol} / \mathrm{mL})$ was pumped $\left(0.125 \mathrm{~mL} \mathrm{~min}^{-1}, t_{\mathrm{R}}=80 \mathrm{~min}\right)$ through a Vaportec UV-150 photochemical reactor $(10 \mathrm{~mL}, \mathrm{FEP}$ tubing) while being irradiated by a $310 \mathrm{~nm}$ UV lamp (output power: $9 \mathrm{~W}$ ) held at $20^{\circ} \mathrm{C}$. The reactor output was monitored using a Mettler Toledo FlowIR instrument ( $\mathrm{SiComp}$ head, bands of interest: $\mathrm{C}=\mathrm{O}$ stretch signal at $1750-1700$ $\mathrm{cm}^{-1}$ for methyl acetate, generated by the decomposition of oxadiazoline). Once the FlowIR detector showed the signal of the reaction slug, the output stream was collected in a sealed sample vial containing a biphasic solution of dichloromethane and brine with stirring to separate excess formaldehyde and other potential impurities. The collected material was rested, and the organic phase was separated and concentrated under reduced pressure. The remaining residue was purified via flash silica gel column chromatography with appropriate eluent combination to give the desired product.

1,2,3,4-Tetrahydronaphthalene-2-carbaldehyde (3a). General Procedure A was followed using 5'-methoxy-5'-methyl-3,4-dihydro$1 \mathrm{H}, 5^{\prime} \mathrm{H}$-spiro(naphthalene-2,2' -[1,3,4] oxadiazole) $(92 \mathrm{mg}, 0.4 \mathrm{mmol}$, 1.0 equiv) and formaldehyde $\left(0.3 \mathrm{~mL}, 37\right.$ wt $\%$ in $\mathrm{H}_{2} \mathrm{O}, 4 \mathrm{mmol}, 10$ equiv). The crude mixture was purified via flash column chromatography (0-20\% EtOAc in petroleum ether) to give the titled product as a transparent oil $(32 \mathrm{mg}, 50 \%) .{ }^{1} \mathrm{H}$ NMR $(600 \mathrm{MHz}$, $\left.\mathrm{CDCl}_{3}\right) \delta 9.80(\mathrm{~d}, J=1.2 \mathrm{~Hz}, 1 \mathrm{H}, \mathrm{HCO}), 7.13(\mathrm{dt}, J=6.3,3.5 \mathrm{~Hz}$, $\left.3 \mathrm{H}, H_{\mathrm{Ar}}\right), 7.10\left(\mathrm{q}, J=4.1,3.5 \mathrm{~Hz}, 1 \mathrm{H}, H_{\mathrm{Ar}}\right), 3.04-2.95(\mathrm{~m}, 2 \mathrm{H}$, 
$\left.\mathrm{ArCH}_{2} \mathrm{CH}_{2}\right), 2.94-2.82\left(\mathrm{~m}, 2 \mathrm{H}, \mathrm{ArCH}_{2}\right), 2.75-2.68$ (m, $1 \mathrm{H}$, $\mathrm{HCOCH}), 2.26-2.19\left(\mathrm{~m}, 1 \mathrm{H}, \mathrm{ArCH}_{a}\right), 1.84-1.75(\mathrm{~m}, 1 \mathrm{H}$, $\left.\mathrm{ArCH}_{a}{ }^{\prime}\right) ;{ }^{13} \mathrm{C}\left\{{ }^{1} \mathrm{H}\right\}$ NMR (151 MHz, $\left.\mathrm{CDCl}_{3}\right) \delta 203.9$ (HCO), 136.1 $\left(C_{\mathrm{Ar}}\right), 134.4\left(C_{\mathrm{Ar}}\right), 129.4\left(C_{\mathrm{Ar}} \mathrm{H}\right), 129.0\left(C_{\mathrm{Ar}} \mathrm{H}\right), 126.2\left(C_{\mathrm{Ar}} \mathrm{H}\right)$, 126.1 $\left(\mathrm{C}_{\mathrm{Ar}} \mathrm{H}\right), 47.0(\mathrm{HCOCH}), 28.6\left(\mathrm{ArCH}_{2} \mathrm{CH}_{2}\right), 28.2\left(\mathrm{ArCH}_{2} \mathrm{CH}\right)$, $23.1\left(C_{\mathrm{a}}\right)$; HRMS (ESI) calcd for $\mathrm{C}_{11} \mathrm{H}_{12} \mathrm{ONa}^{+}[\mathrm{M}+\mathrm{Na}]^{+}$183.0780, found 183.0775; IR $\nu_{\max }$ (film) 2904, 2851, 1723, 1702, 1432, 1110, $1042 \mathrm{~cm}^{-1}$. The data presented are consistent with literature precedent. ${ }^{29}$

Tetrahydro-2H-pyran-4-carbaldehyde (3b). General procedure A was followed using 3-methoxy-3-methyl-4,8-dioxa-1,2-diazaspiro[4.5]dec-1-ene ( $74 \mathrm{mg}, 0.4 \mathrm{mmol}, 1.0$ equiv) and formaldehyde $(0.3 \mathrm{~mL}$, 37 wt $\%$ in $\mathrm{H}_{2} \mathrm{O}, 4 \mathrm{mmol}, 10$ equiv). The crude mixture was purified via flash column chromatography (0-15\% EtOAc in petroleum ether) to give the titled product as a volatile transparent oil $(23 \mathrm{mg}, 48 \%)$ : ${ }^{1} \mathrm{H}$ NMR $\left(600 \mathrm{MHz}, \mathrm{CDCl}_{3}\right) \delta 9.68(\mathrm{~d}, J=1.0 \mathrm{~Hz}, 1 \mathrm{H}, \mathrm{HCO}), 4.00-$ $3.92\left(\mathrm{~m}, 2 \mathrm{H}, \mathrm{OCH}_{\mathrm{a}}+\mathrm{OCH}_{\mathrm{b}}\right), 3.48(\mathrm{ddd}, J=11.5,10.7,2.6 \mathrm{~Hz}, 2 \mathrm{H}$, $\left.\mathrm{OCH}_{\mathrm{a}}{ }^{\prime}+\mathrm{OCH}_{\mathrm{b}}{ }^{\prime \prime}\right), 2.55-2.36(\mathrm{~m}, 1 \mathrm{H}, \mathrm{HCOCH}), 1.89-1.83(\mathrm{~m}, 2 \mathrm{H}$, $\left.\mathrm{OCH}_{\mathrm{c}}+\mathrm{OCH}_{\mathrm{d}}\right), 1.70\left(\mathrm{dtd}, J=13.7,10.7,4.2 \mathrm{~Hz}, 2 \mathrm{H}, \mathrm{OCH}_{\mathrm{c}}{ }^{\prime}+\right.$ $\left.\mathrm{OCH}_{\mathrm{d}}{ }^{\prime}\right) ;{ }^{13} \mathrm{C}\left\{{ }^{1} \mathrm{H}\right\}$ NMR (151 MHz, $\left.\mathrm{CDCl}_{3}\right) \delta 203.0$ (HCO), 66.8 $\left(C_{\mathrm{a}}+C_{\mathrm{b}}\right), 46.9(\mathrm{HCOCH}), 25.8\left(C_{\mathrm{c}}+C_{\mathrm{d}}\right) ;$ LRMS $($ ESI, $m / z) 115.2$ $\left([\mathrm{M}+\mathrm{H}]^{+}, 100\right)$; IR $\nu_{\max }$ (film) 2968, 1879, 1720, 1279, 1201, 1135, $1080,924 \mathrm{~cm}^{-1}$. The data presented are consistent with literature precedent. ${ }^{30}$

Tetrahydro-2H-thiopyran-4-carbaldehyde (3c). General procedure A was followed using 3-methoxy-3-methyl-4-oxa-8-thia-1,2diazaspiro[4.5] dec-1-ene ( $81 \mathrm{mg}, 0.4 \mathrm{mmol}, 1.0$ equiv) and formaldehyde $\left(0.3 \mathrm{~mL}, 37 \mathrm{wt} \%\right.$ in $\mathrm{H}_{2} \mathrm{O}, 4 \mathrm{mmol}, 10$ equiv). The crude mixture was purified via flash column chromatography (0-20\% EtOAc in petroleum ether) to give the titled product as a volatile transparent oil (29 mg, 56\%): ${ }^{1} \mathrm{H}$ NMR $\left(600 \mathrm{MHz}, \mathrm{CDCl}_{3}\right) \delta 9.59(\mathrm{~s}$, $1 \mathrm{H}, \mathrm{HCO}), 2.67\left(\mathrm{dt}, J=10.2,3.6 \mathrm{~Hz}, 4 \mathrm{H}, \mathrm{SCH}_{2}\right), 2.34-2.22(\mathrm{~m}, 3 \mathrm{H}$, $\left.\mathrm{HCOCH}+\mathrm{CH}_{\mathrm{a}}+\mathrm{CH}_{\mathrm{b}}\right), 1.75\left(\mathrm{dtd}, J=14.3,10.2,4.5 \mathrm{~Hz}, 2 \mathrm{H}, \mathrm{CH}_{\mathrm{a}}{ }^{\prime}+\right.$ $\left.\mathrm{CH}_{\mathrm{b}}{ }^{\prime}\right) ;{ }^{13} \mathrm{C}\left\{{ }^{1} \mathrm{H}\right\}$ NMR $\left(151 \mathrm{MHz}, \mathrm{CDCl}_{3}\right) \delta 203.3$ (HCO), 49.3 $(\mathrm{HCOCH}), 27.6\left(\mathrm{SCH}_{2}\right), 27.2\left(\mathrm{C}_{\mathrm{a}}+\mathrm{C}_{\mathrm{b}}\right)$; HRMS (ESI) calcd for $\mathrm{C}_{6} \mathrm{H}_{11} \mathrm{OS}^{+}[\mathrm{M}+\mathrm{H}]^{+}$131.0528, found 131.0531; IR $\nu_{\max }$ (film) 2918, $2849,2369,1724,1239,1130,1088,983 \mathrm{~cm}^{-1}$. The data presented are consistent with literature precedent. ${ }^{31}$

Tetrahydrothiophene-3-carbaldehyde (3d). General procedure A was followed using 3-methoxy-3-methyl-4-oxa-7-thia-1,2diazaspiro[4.4]non-1-ene (37 mg, $0.2 \mathrm{mmol}, 1.0$ equiv) and formaldehyde ( $0.16 \mathrm{~mL}, 37$ wt $\%$ in $\mathrm{H}_{2} \mathrm{O}, 2.0 \mathrm{mmol}, 10$ equiv). An 85\% NMR yield was calculated using 1,3,5-trimethoxybenzene (11 $\mathrm{mg}, 0.066 \mathrm{mmol}, 0.33$ equiv) as an internal standard. The crude mixture was purified via flash column chromatography (0-20\% EtOAc in petroleum ether) to give the titled product as a colorless volatile oil $(17 \mathrm{mg}, 75 \%)$ with less than $10 \%$ of dichloromethane: ${ }^{1} \mathrm{H}$ $\operatorname{NMR}\left(600 \mathrm{MHz}, \mathrm{CDCl}_{3}\right) \delta 9.63(\mathrm{~d}, J=1.3 \mathrm{~Hz}, 1 \mathrm{H}, \mathrm{HCO}), 3.16$ (dd, $\left.J=10.9,5.0 \mathrm{~Hz}, 1 \mathrm{H}, \mathrm{SCH}_{\mathrm{c}} \mathrm{CH}\right), 3.09-3.04(\mathrm{~m}, 1 \mathrm{H}, \mathrm{HCOCH}), 2.98$ (dd, $\left.J=10.9,7.0 \mathrm{~Hz}, 1 \mathrm{H}, \mathrm{SCH}_{\mathrm{c}} \mathrm{CH}\right), 2.91-2.85\left(\mathrm{~m}, 1 \mathrm{H}, \mathrm{SCH}_{\mathrm{b}} \mathrm{CH}_{2}\right)$, $2.85-2.78\left(\mathrm{~m}, 1 \mathrm{H}, \mathrm{SCH}_{\mathrm{b}} \mathrm{CH}_{2}\right), 2.38(\mathrm{td}, J=12.7,5.9 \mathrm{~Hz}, 1 \mathrm{H}$, $\left.\mathrm{CHCH}_{\mathrm{a}}\right), 2.12\left(\mathrm{dq}, J=13.4,6.9 \mathrm{~Hz}, 1 \mathrm{H}, \mathrm{CHCH}_{\mathrm{a}}{ }^{\prime}\right) ;{ }^{13} \mathrm{C}\left\{{ }^{1} \mathrm{H}\right\} \mathrm{NMR}$ $\left(151 \mathrm{MHz}, \mathrm{CDCl}_{3}\right) \delta 201.3(\mathrm{HCO}), 55.1(\mathrm{HCOCH}), 31.1\left(C_{\mathrm{c}}\right), 30.9$ $\left(C_{\mathrm{b}}\right), 30.5\left(C_{\mathrm{a}}\right)$; LRMS (ESI, $\left.m / z\right) 117.1\left([\mathrm{M}+\mathrm{H}]^{+}, 100\right)$; IR $\nu_{\max }$ (film) 2944, 1720,1416,1235,1028, $956 \mathrm{~cm}^{-1}$. The data presented are consistent with literature precedent. ${ }^{32}$

1,4-Dioxaspiro[4.5]decane-8-carbaldehyde (3e). General procedure A was followed using 3-methoxy-3-methyl-4,9,12-trioxa-1,2diazadispiro $\left[4.2 .4^{8} .2^{5}\right]$ tetradec-1-ene $(102 \mathrm{mg}, 0.4 \mathrm{mmol}, 1.0$ equiv) and formaldehyde $\left(0.3 \mathrm{~mL}, 37 \mathrm{wt} \%\right.$ in $\mathrm{H}_{2} \mathrm{O}, 4 \mathrm{mmol}, 10$ equiv). The crude mixture was purified via flash column chromatography $(0-20 \%$ EtOAc in petroleum ether) to give the titled product as a colorless volatile oil (44 mg, $65 \%):{ }^{1} \mathrm{H} \mathrm{NMR}\left(600 \mathrm{MHz}, \mathrm{CDCl}_{3}\right) \delta 9.64(\mathrm{~d}, J=$ $1.3 \mathrm{~Hz}, 1 \mathrm{H}, \mathrm{HCO}), 3.94\left(\mathrm{dd}, J=5.3,3.4 \mathrm{~Hz}, 4 \mathrm{H}, \mathrm{OCH}_{2} \mathrm{CH}_{2} \mathrm{O}\right), 2.25$ (ttd, $J=9.7,4.1,1.4 \mathrm{~Hz}, 1 \mathrm{H}, \mathrm{HCOCH}), 1.97-1.91\left(\mathrm{~m}, 2 \mathrm{H}, \mathrm{CH}_{\mathrm{c}}+\right.$ $\left.\mathrm{CH}_{\mathrm{d}}\right), 1.80-1.71\left(\mathrm{~m}, 4 \mathrm{H}, \mathrm{CH}_{\mathrm{c}}{ }^{\prime}+\mathrm{CH}_{\mathrm{d}}{ }^{\prime}+\mathrm{CH}_{\mathrm{e}}+\mathrm{CH}_{\mathrm{f}}\right), 1.61-1.56(\mathrm{~m}$, $\left.2 \mathrm{H}, \mathrm{CH}_{\mathrm{e}}+\mathrm{CH}_{\mathrm{f}}\right) ;{ }^{13} \mathrm{C}\left\{{ }^{1} \mathrm{H}\right\} \mathrm{NMR}\left(151 \mathrm{MHz}, \mathrm{CDCl}_{3}\right) \delta 204.2(\mathrm{HCO})$, 108.2 (OCO), $64.5\left(C_{\mathrm{a}}\right), 64.5\left(C_{\mathrm{b}}\right), 48.4(\mathrm{HCOCH}), 33.5\left(C_{\mathrm{c}}+C_{\mathrm{d}}\right)$, $23.4\left(C_{\mathrm{e}}+C_{\mathrm{f}}\right)$; LRMS (ESI, $\left.m / z\right) 171.4\left([\mathrm{M}+\mathrm{H}]^{+}, 100\right)$; IR $\nu_{\max }$ (film) 2949, 2881, 1722, 1447, 1362, 1239, 1142, 1104, 1033, 948,
$881 \mathrm{~cm}^{-1}$. The data presented are consistent with literature precedent. $^{33}$

tert-Butyl 4-Formylpiperidine-1-carboxylate (3f). General procedure A was followed using tert-butyl 3-methoxy-3-methyl-4-oxa-1,2,8triazaspiro[4.5] dec-1-ene- 8 -carboxylate $(57 \mathrm{mg}, 0.2 \mathrm{mmol}, 1.0$ equiv) and formaldehyde $\left(0.16 \mathrm{~mL}, 37 \mathrm{wt} \%\right.$ in $\mathrm{H}_{2} \mathrm{O}, 2.0 \mathrm{mmol}, 10$ equiv). A $58 \%$ NMR yield was calculated using 1,3,5-trimethoxybenzene (11 $\mathrm{mg}, 0.066 \mathrm{mmol}, 0.33$ equiv) as an internal standard. The crude mixture was purified via flash column chromatography (0-20\% EtOAc in petroleum ether) to give the titled product as a colorless oil (21 mg, 49\%): ${ }^{1} \mathrm{H}$ NMR (600 MHz, $\left.\mathrm{CDCl}_{3}\right) \delta 9.65$ (s, 1H, HCO), $3.97\left(\right.$ br s, $\left.2 \mathrm{H}, \mathrm{NCH}_{\mathrm{c}}+\mathrm{NCH}_{\mathrm{d}}\right), 3.02-2.81\left(\mathrm{~m}, 2 \mathrm{H}, \mathrm{NCH}_{\mathrm{c}}{ }^{\prime}+\right.$ $\left.\mathrm{NCH}_{\mathrm{d}}{ }^{\prime}\right), 2.47-2.36(\mathrm{~m}, 1 \mathrm{H}, \mathrm{HCOCH}), 1.98-1.80\left(\mathrm{~m}, 2 \mathrm{H}, \mathrm{CH}_{\mathrm{a}}+\right.$ $\left.\mathrm{CH}_{\mathrm{b}}\right), 1.59-1.50\left(\mathrm{~m}, 2 \mathrm{H}, \mathrm{CH}_{\mathrm{a}}{ }^{\prime}+\mathrm{CH}_{\mathrm{b}}{ }^{\prime}\right), 1.44\left(\mathrm{~s}, 9 \mathrm{H}, \mathrm{C}\left(\mathrm{CH}_{3}\right)_{3}\right)$; ${ }^{13} \mathrm{C}\left\{{ }^{1} \mathrm{H}\right\}$ NMR $\left(151 \mathrm{MHz}, \mathrm{CDCl}_{3}\right) \delta 203.1$ (HCO), 154.8 (NCOO), $79.8\left(\mathrm{C}\left(\mathrm{CH}_{3}\right)_{3}\right), 48.1(\mathrm{HCOCH}), 43.0\left(\mathrm{br}, \mathrm{C}_{\mathrm{c}}+\mathrm{C}_{\mathrm{d}}\right), 28.5$ $\left(\mathrm{C}\left(\mathrm{CH}_{3}\right)_{3}\right), 25.3\left(\mathrm{C}_{\mathrm{a}}+\mathrm{C}_{\mathrm{b}}\right)$; LRMS (ESI, $\left.m / z\right) 214.3\left([\mathrm{M}+\mathrm{H}]^{+}\right.$, 100); IR $\nu_{\max }$ (film) 2927, 1726, 1688, 1418, 1365, 1273, 1232, 1168, $1128,958,864,769 \mathrm{~cm}^{-1}$. The data presented are consistent with literature precedent. ${ }^{34}$

Adamantane-2-carbaldehyde (3h). General procedure A was followed using $5^{\prime}$-methoxy-5'-methyl-5' $H$-spiro[adamantane-2, $2^{\prime}$ $[1,3,4]$ oxadiazole] ( $94 \mathrm{mg}, 0.4 \mathrm{mmol}, 1.0$ equiv) and formaldehyde ( $0.3 \mathrm{~mL}, 37 \mathrm{wt} \%$ in $\mathrm{H}_{2} \mathrm{O}, 4 \mathrm{mmol}, 10$ equiv). The crude mixture was purified via flash column chromatography $(0-20 \%$ EtOAc in petroleum ether) to give the titled product as a white solid $(45 \mathrm{mg}$, $68 \%):{ }^{1} \mathrm{H}$ NMR $\left(600 \mathrm{MHz}, \mathrm{CDCl}_{3}\right) \delta 9.73$ (s, 1H, HCO), 2.44-2.37 $(\mathrm{m}, 3 \mathrm{H}, \mathrm{HCOCH}+\mathrm{HCOCHCH}), 2.01-1.67\left(\mathrm{~m}, 12 \mathrm{H}, \mathrm{CH}_{\mathrm{b}}+\mathrm{CH}_{\mathrm{c}}\right.$ $\left.+\mathrm{CH}_{\mathrm{d}}\right) ;{ }^{13} \mathrm{C}\left\{{ }^{1} \mathrm{H}\right\}$ NMR $\left(151 \mathrm{MHz}, \mathrm{CDCl}_{3}\right) \delta 206.1$ (HCO), 56.7 $(\mathrm{HCOCH}), 38.0\left(C_{\mathrm{b}}\right), 37.2\left(C_{\mathrm{d}}\right), 33.7\left(C_{\mathrm{b}}{ }^{\prime}\right), 28.3\left(C_{\mathrm{a}}\right), 28.0\left(C_{\mathrm{c}}\right)$, $27.6\left(C_{\mathrm{c}}{ }^{\prime}\right)$; HRMS (ESI) calcd for $\mathrm{C}_{11} \mathrm{H}_{17} \mathrm{O}^{+}[\mathrm{M}+\mathrm{H}]^{+}$165.1274, found 165.1271; IR $\nu_{\max }$ (film) 2936, 2896, 1752, 1463, 1190, 1076, $912 \mathrm{~cm}^{-1}$; mp $164-166{ }^{\circ} \mathrm{C}$. The data presented are consistent with literature precedent. $^{35}$

4-(6-Methoxynaphthalen-2-yl)-2-methylbutanal (3j). General procedure A was followed using 2-methoxy-5-(2-(6-methoxynaphthalen-2-yl)ethyl)-2,5-dimethyl-2,5-dihydro-1,3,4-oxadiazole (126 mg, $0.4 \mathrm{mmol}, 1.0$ equiv) and formaldehyde $\left(0.3 \mathrm{~mL}, 37 \mathrm{wt} \%\right.$ in $\mathrm{H}_{2} \mathrm{O}, 4$ mmol, 10 equiv). The crude mixture was purified via flash column chromatography $(0-20 \%$ EtOAc in petroleum ether) to give the titled product as a transparent oil $(55 \mathrm{mg}, 57 \%)$ together with $7 \%$ of oxidized carboxylic acid: ${ }^{1} \mathrm{H}$ NMR $\left(600 \mathrm{MHz}, \mathrm{CDCl}_{3}\right) \delta 9.65(\mathrm{~d}, J=$ $1.8 \mathrm{~Hz}, 1 \mathrm{H}, H C O), 7.68\left(\mathrm{dd}, J=8.5,3.4 \mathrm{~Hz}, 2 \mathrm{H}, H_{\mathrm{Ar}}\right), 7.55(\mathrm{~d}, J=$ $\left.1.8 \mathrm{~Hz}, 1 \mathrm{H}, H_{\mathrm{Ar}}\right), 7.30\left(\mathrm{dd}, J=8.4,1.8 \mathrm{~Hz}, 1 \mathrm{H}, H_{\mathrm{Ar}}\right), 7.16-7.10(\mathrm{~m}$, $\left.2 \mathrm{H}, H_{\mathrm{Ar}}\right), 3.92\left(\mathrm{~s}, 3 \mathrm{H}, \mathrm{OCH}_{3}\right), 2.88-2.75(\mathrm{~m}, 2 \mathrm{H}, \mathrm{HCOCH}+$ $\mathrm{ArCH}_{\mathrm{a}}$ ), $2.41\left(\mathrm{qd}, J=6.9,1.8 \mathrm{~Hz}, 1 \mathrm{H}, \mathrm{ArCH}_{\mathrm{a}}{ }^{\prime}\right), 2.19-2.10(\mathrm{~m}, 1 \mathrm{H}$, $\mathrm{ArCH}_{2} \mathrm{CH}_{\mathrm{b}}$ ), $1.78-1.70\left(\mathrm{~m}, 1 \mathrm{H}, \mathrm{ArCH}_{2} \mathrm{CH}_{\mathrm{b}}{ }^{\prime}\right), 1.18(\mathrm{~d}, J=7.1 \mathrm{~Hz}$, $\left.3 \mathrm{H}, \mathrm{CHCH}_{3}\right) ;{ }^{13} \mathrm{C}\left\{{ }^{1} \mathrm{H}\right\}$ NMR (151 MHz, $\left.\mathrm{CDCl}_{3}\right) \delta 205.0(\mathrm{HCO})$, $157.4\left(C_{\mathrm{Ar}}\right), 136.6\left(C_{\mathrm{Ar}}\right), 133.2\left(C_{\mathrm{Ar}}\right), 129.2\left(C_{\mathrm{Ar}}\right), 129.0\left(C_{\mathrm{Ar}} \mathrm{H}\right)$, $127.7\left(C_{\mathrm{Ar}} \mathrm{H}\right), 127.1\left(C_{\mathrm{Ar}} \mathrm{H}\right), 126.5\left(C_{\mathrm{Ar}} \mathrm{H}\right), 119.0\left(C_{\mathrm{Ar}} \mathrm{H}\right), 105.8$ $\left(C_{\mathrm{Ar}} \mathrm{H}\right), 55.3\left(\mathrm{OCH}_{3}\right), 45.6(\mathrm{HCOCH}), 33.0\left(C_{\mathrm{a}}\right), 32.1\left(C_{\mathrm{b}}\right), 13.4$ $\left(\mathrm{CHCH}_{3}\right)$; HRMS (ESI) calcd for $\mathrm{C}_{16} \mathrm{H}_{19} \mathrm{O}_{2}{ }^{+}[\mathrm{M}+\mathrm{H}]^{+}$243.1385, found 243.1386; IR $\nu_{\max }$ (film) 2933, 1721, 1634, 1606, 1483, 1390, $1264,1229,1031,850 \mathrm{~cm}^{-1}$.

Synthesis of Alcohols. General Procedure B for the Synthesis of Alcohols. The reaction slug from general procedure A was directly collected into a round-bottom flask containing $\mathrm{NaBH}_{4}$ (10 equiv) in EtOH $(0.5 \mathrm{mmol} / \mathrm{mL})$ and stirred for a further $1 \mathrm{~h}$. The resulting mixture was then quenched with ice-water, extracted with ethyl acetate $(2 \times 20 \mathrm{~mL})$, and washed with brine $(2 \times 20 \mathrm{~mL})$. The organic phase was combined, dried over $\mathrm{MgSO}_{4}$, filtered, and concentrated under reduced pressure. The remaining residue was purified via flash column chromatography with appropriate eluents to give the desired alcohol.

(1,2,3,4-Tetrahydronaphthalen-2-yl)methanol (4a). General procedure $\mathrm{B}$ was followed using $5^{\prime}$-methoxy-5'-methyl-3,4-dihydro$1 H, 5^{\prime} H$-spiro[naphthalene-2,2' $-[1,3,4]$ oxadiazole ] (92 mg, 0.4 mmol, 1.0 equiv), formaldehyde $\left(0.3 \mathrm{~mL}, 37\right.$ wt $\%$ in $\mathrm{H}_{2} \mathrm{O}, 4$ mmol, 10 equiv), and sodium borohydride $(153 \mathrm{mg}, 4.0 \mathrm{mmol}, 10$ equiv). The crude mixture was purified via flash column 
chromatography (10-40\% EtOAc in petroleum ether) to give the titled product as a transparent oil $(39 \mathrm{mg}, 60 \%):{ }^{1} \mathrm{H}$ NMR $(600 \mathrm{MHz}$, $\mathrm{CDCl}_{3}$ ) $\delta 7.09$ (app. p, $J=2.2 \mathrm{~Hz}, 4 \mathrm{H}, \mathrm{H}_{\mathrm{Ar}}$ ), 3.69-3.59 (m, $2 \mathrm{H}$, $\left.\mathrm{HOCH}_{2}\right), 2.93-2.79\left(\mathrm{~m}, 3 \mathrm{H}, \mathrm{ArCH}_{2} \mathrm{CH}+\mathrm{ArCH}_{\mathrm{a}}\right), 2.52(\mathrm{dd}, J=$ $\left.16.3,10.7 \mathrm{~Hz}, 1 \mathrm{H}, \mathrm{ArCH}_{\mathrm{a}}{ }^{\prime}\right), 2.06-1.95\left(\mathrm{~m}, 2 \mathrm{H}, \mathrm{ArCH}_{2} \mathrm{CH}_{2}\right), 1.55-$ $1.39\left(\mathrm{~m}, 2 \mathrm{H}, \mathrm{HOCH}_{2} \mathrm{CH}+\mathrm{CH}_{2} \mathrm{OH}\right) ;{ }^{13} \mathrm{C}\left\{{ }^{1} \mathrm{H}\right\}$ NMR $(151 \mathrm{MHz}$, $\left.\mathrm{CDCl}_{3}\right) \delta 136.8\left(C_{\mathrm{Ar}}\right), 136.0\left(C_{\mathrm{Ar}}\right), 129.3\left(C_{\mathrm{Ar}} \mathrm{H}\right), 128.9\left(C_{\mathrm{Ar}} \mathrm{H}\right)$, $125.7\left(\mathrm{C}_{\mathrm{Ar}} \mathrm{H}\right), 125.7\left(\mathrm{C}_{\mathrm{Ar}} \mathrm{H}\right), 67.8\left(\mathrm{HOCH}_{2}\right), 37.1\left(\mathrm{HOCH}_{2} \mathrm{CH}\right)$, $32.5\left(\mathrm{ArCH}_{2}\right), 28.8\left(\mathrm{C}_{\mathrm{a}}\right), 26.0\left(\mathrm{ArCH}_{2} \mathrm{CH}_{2}\right)$; HRMS (ESI) calcd for $\mathrm{C}_{11} \mathrm{H}_{14} \mathrm{Ona}^{+}[\mathrm{M}+\mathrm{Na}]^{+} 185.0937$, found 185.0931; IR $\nu_{\max }$ (film) $3370,2918,1494,1453,1436,1065,1022,900 \mathrm{~cm}^{-1}$. The data presented are consistent with literature precedent. ${ }^{36}$

(Tetrahydro-2H-pyran-4-yl)methanol (4b). General procedure B was followed using 3-methoxy-3-methyl-4,8-dioxa-1,2-diazaspiro[4.5]dec-1-ene ( $74 \mathrm{mg}, 0.4 \mathrm{mmol}, 1.0$ equiv), formaldehyde $(0.3 \mathrm{~mL}, 37 \mathrm{wt}$ $\%$ in $\mathrm{H}_{2} \mathrm{O}, 4 \mathrm{mmol}, 10$ equiv), and sodium borohydride ( $153 \mathrm{mg}, 4.0$ mmol, 10 equiv). The crude mixture was purified via flash column chromatography (10-40\% EtOAc in petroleum ether) to give the titled product as a transparent oil $(25 \mathrm{mg}, 53 \%):{ }^{1} \mathrm{H}$ NMR $(600 \mathrm{MHz}$, $\left.\mathrm{CDCl}_{3}\right) \delta 3.99\left(\mathrm{ddt}, J=11.5,4.6,1.1 \mathrm{~Hz}, 2 \mathrm{H}, \mathrm{OCH}_{\mathrm{a}}+\mathrm{OCH}_{\mathrm{b}}\right), 3.51$ $\left(\mathrm{d}, J=6.5 \mathrm{~Hz}, 2 \mathrm{H}, \mathrm{HOCH}_{2}\right), 3.41\left(\mathrm{td}, J=11.5,2.1 \mathrm{~Hz}, 2 \mathrm{H}, \mathrm{OCH}_{\mathrm{a}}{ }^{\prime}+\right.$ $\left.\mathrm{OCH}_{\mathrm{b}}{ }^{\prime}\right), 1.79-1.73\left(\mathrm{~m}, 1 \mathrm{H}, \mathrm{HOCH}_{2} \mathrm{CH}\right), 1.68-1.64\left(\mathrm{~m}, 2 \mathrm{H}, \mathrm{CH}_{\mathrm{c}}+\right.$ $\left.\mathrm{CH}_{\mathrm{d}}\right), 1.38-1.32\left(\mathrm{~m}, 2 \mathrm{H}, \mathrm{CH}_{\mathrm{c}}{ }^{\prime}+\mathrm{CH}_{\mathrm{d}}{ }^{\prime}\right) ;{ }^{13} \mathrm{C}\left\{{ }^{1} \mathrm{H}\right\}$ NMR $(151 \mathrm{MHz}$, $\left.\mathrm{CDCl}_{3}\right) \delta 68.1\left(\mathrm{HOCH}_{2}\right), 67.8\left(\mathrm{C}_{\mathrm{a}}+\mathrm{C}_{\mathrm{b}}\right), 37.7\left(\mathrm{HOCH}_{2} \mathrm{CH}\right), 29.4$ $\left(C_{\mathrm{c}}+C_{\mathrm{d}}\right)$; LRMS (ESI, $\left.m / z\right) 115.3\left([\mathrm{M}-\mathrm{H}]^{-}, 100\right) ; \operatorname{IR} \nu_{\max }($ film $)$ $3368,2918,2847,1652,1443,1235,1140,1031,1012,984,849$ $\mathrm{cm}^{-1}$. The data presented are consistent with literature precedent. ${ }^{30}$

(Tetrahydro-2H-thiopyran-4-yl)methanol (4c). General procedure $\mathrm{B}$ was followed using 3-methoxy-3-methyl-4-oxa-8-thia-1,2diazaspiro[4.5] dec-1-ene ( $81 \mathrm{mg}, 0.4 \mathrm{mmol}, 1.0$ equiv), formaldehyde (0.3 mL, 37 wt $\%$ in $\mathrm{H}_{2} \mathrm{O}, 4 \mathrm{mmol}, 10$ equiv), and sodium borohydride ( $153 \mathrm{mg}, 4.0 \mathrm{mmol}, 10$ equiv). The crude mixture was purified via flash column chromatography $(10-40 \%$ EtOAc in petroleum ether) to give the titled product as a transparent oil (38 mg, 72\%): ${ }^{1} \mathrm{H}$ NMR $\left(600 \mathrm{MHz}, \mathrm{CDCl}_{3}\right) \delta 3.47(\mathrm{~d}, J=6.4 \mathrm{~Hz}, 2 \mathrm{H}$, $\left.\mathrm{HOCH}_{2}\right), 2.70\left(\mathrm{ddd}, J=14.3,11.9,2.6 \mathrm{~Hz}, 2 \mathrm{H}, \mathrm{SCH}_{\mathrm{a}}+\mathrm{SCH}_{\mathrm{b}}\right)$, 2.64-2.58 (m, 2H, $\left.\mathrm{SCH}_{\mathrm{a}}{ }^{\prime}+\mathrm{SCH}_{\mathrm{b}}{ }^{\prime}\right), 2.07(\mathrm{dd}, J=13.5,3.5 \mathrm{~Hz}, 2 \mathrm{H}$, $\left.\mathrm{CH}_{\mathrm{c}}+\mathrm{CH}_{\mathrm{d}}\right), 1.59($ br s, $1 \mathrm{H}, \mathrm{OH}), 1.57-1.48\left(\mathrm{~m}, 1 \mathrm{H}, \mathrm{HOCH}_{2} \mathrm{CH}\right)$, $1.39\left(\mathrm{dtd}, J=13.1,11.8,3.5 \mathrm{~Hz}, 1 \mathrm{H}, \mathrm{CH}_{\mathrm{c}}{ }^{\prime}+\mathrm{CH}_{\mathrm{d}}{ }^{\prime}\right) ;{ }^{13} \mathrm{C}\left\{{ }^{1} \mathrm{H}\right\} \mathrm{NMR}$ $\left(151 \mathrm{MHz}, \mathrm{CDCl}_{3}\right) \delta 68.4\left(\mathrm{HOCH}_{2}\right), 40.2\left(\mathrm{HOCH}_{2} \mathrm{CH}\right), 30.8\left(\mathrm{C}_{\mathrm{a}}+\right.$ $\left.C_{\mathrm{b}}\right), 28.3\left(C_{\mathrm{c}}+C_{\mathrm{d}}\right)$; HRMS (ESI) calcd for $\mathrm{C}_{6} \mathrm{H}_{13} \mathrm{O}^{32} \mathrm{~S}^{+}[\mathrm{M}+\mathrm{H}]^{+}$ 133.0682, found 133.0681; IR $\nu_{\max }$ (film) 3584, 2924, 1454, 1422, $1273,1036 \mathrm{~cm}^{-1}$. The data presented are consistent with literature precedent. ${ }^{37}$

(Tetrahydrothiophene-3-yl)methanol (4d). General procedure B was followed using 3-methoxy-3-methyl-4-oxa-7-thia-1,2diazaspiro[4.4]non-1-ene ( $37 \mathrm{mg}, 0.2 \mathrm{mmol}, 1.0$ equiv), formaldehyde (0.16 mL, 37 wt \% in $\mathrm{H}_{2} \mathrm{O}, 2.0 \mathrm{mmol}, 10$ equiv), and sodium borohydride (76 mg, $2.0 \mathrm{mmol}, 10$ equiv). An 89\% NMR yield was calculated using 1,3,5-trimethoxybenzene $(11 \mathrm{mg}, 0.066 \mathrm{mmol}, 0.33$ equiv) as an internal standard. The crude mixture was purified via flash column chromatography (0-40\% EtOAc in petroleum ether) to give the titled product as a colorless oil $(18 \mathrm{mg}, 77 \%)$ with less than $5 \%$ of ethyl acetate: ${ }^{1} \mathrm{H}$ NMR $\left(600 \mathrm{MHz}, \mathrm{CDCl}_{3}\right) \delta 3.64(\mathrm{dt}, J=6.8$, $\left.3.5 \mathrm{~Hz}, 2 \mathrm{H}, \mathrm{HOCH}_{2}\right), 2.94\left(\mathrm{dd}, J=10.6,6.8 \mathrm{~Hz}, 1 \mathrm{H}, \mathrm{SCH}_{\mathrm{c}} \mathrm{CH}\right), 2.87$ (ddd, $\left.J=7.2,5.9,1.6 \mathrm{~Hz}, 2 \mathrm{H}, \mathrm{SCH}_{\mathrm{b}} \mathrm{CH}_{2}+\mathrm{SCH}_{\mathrm{b}}{ }^{\prime} \mathrm{CH}_{2}\right), 2.65$ (dd, $J=$ 10.6, 7.2 Hz, $\left.1 \mathrm{H}, \mathrm{SCH}_{\mathrm{c}}{ }^{\prime} \mathrm{CH}\right), 2.44$ (dpd, $J=8.3,6.8,5.5 \mathrm{~Hz}, 1 \mathrm{H}$, $\left.\mathrm{HOCH}_{2} \mathrm{CH}\right), 2.12\left(\mathrm{dq}, J=11.9,5.7 \mathrm{~Hz}, 1 \mathrm{H}, \mathrm{CHCH}_{\mathrm{a}}\right), 1.85-1.71(\mathrm{~m}$, $\left.2 \mathrm{H}, \mathrm{OH}+\mathrm{CHCH}_{\mathrm{a}}{ }^{\prime}\right) ;{ }^{13} \mathrm{C}\left\{{ }^{1} \mathrm{H}\right\} \operatorname{NMR}\left(151 \mathrm{MHz}, \mathrm{CDCl}_{3}\right) \delta 64.8$ $\left(\mathrm{HOCH}_{2}\right), 46.7\left(\mathrm{HOCH}_{2} \mathrm{CH}\right), 33.8\left(C_{\mathrm{c}}\right), 33.4\left(C_{\mathrm{d}}\right), 30.9\left(\mathrm{C}_{\mathrm{a}}\right)$; LRMS (ESI, $m / z) 117.3$ ([M - H $]^{-}$, 100); IR $\nu_{\max }$ (film) 3336, 2928, $2860,2355,1438,1264,1210,1079,1049,1028,967,945,885,684$ $\mathrm{cm}^{-1}$. The data presented are consistent with literature precedent. ${ }^{38}$

(1,4-Dioxaspiro[4.5]decan-8-yl)metanol (4e). General procedure $B$ was followed using 3-methoxy-3-methyl-4,9,12-trioxa-1,2diazadispiro $\left[4.2 .4^{8} .2^{5}\right]$ tetradec-1-ene $(102 \mathrm{mg}, 0.4 \mathrm{mmol}, 1.0$ equiv), formaldehyde ( $0.3 \mathrm{~mL}, 37 \mathrm{wt} \%$ in $\mathrm{H}_{2} \mathrm{O}, 4 \mathrm{mmol}, 10$ equiv), and sodium borohydride (152 mg, $4.0 \mathrm{mmol}, 10$ equiv). The crude mixture was purified via flash column chromatography (10-40\% EtOAc in petroleum ether) to give the titled product as a transparent oil (52 mg, 75\%): ${ }^{1} \mathrm{H}$ NMR (600 MHz, $\left.\mathrm{CDCl}_{3}\right) \delta 4.09-3.78(\mathrm{~m}, 4 \mathrm{H}$, $\mathrm{OCH}_{2} \mathrm{CH}_{2} \mathrm{O}$ ), $3.46\left(\mathrm{~d}, J=6.5 \mathrm{~Hz}, 2 \mathrm{H}, \mathrm{HOCH}_{2}\right), 1.86($ br s, $1 \mathrm{H}$, $\mathrm{HO}), 1.78-1.73\left(\mathrm{~m}, 4 \mathrm{H}, \mathrm{CH}_{\mathrm{c}}+\mathrm{CH}_{\mathrm{d}}\right), 1.52(\mathrm{td}, J=13.5,12.8,4.6$ $\left.\mathrm{Hz}, 3 \mathrm{H}, \mathrm{HOCH}_{2} \mathrm{CH}+\mathrm{OCCH}_{\mathrm{a}}+\mathrm{OCCH}_{\mathrm{b}}\right), 1.26(\mathrm{dtd}, J=13.5,12.8$, $\left.11.7,4.0 \mathrm{~Hz}, 2 \mathrm{H}, \mathrm{OCCH}_{\mathrm{a}}{ }^{\prime}+\mathrm{OCCH}_{\mathrm{b}}{ }^{\prime}\right) ;{ }^{13} \mathrm{C}\left\{{ }^{1} \mathrm{H}\right\} \mathrm{NMR}(151 \mathrm{MHz}$, $\left.\mathrm{CDCl}_{3}\right) \delta 109.1(\mathrm{OCO}), 67.8\left(\mathrm{HOCH}_{2}\right), 64.2\left(\mathrm{OCH}_{2} \mathrm{CH}_{2} \mathrm{O}\right), 39.2$ $\left(\mathrm{HOCH}_{2} \mathrm{CH}\right), 34.2\left(\mathrm{C}_{\mathrm{a}}+\mathrm{C}_{\mathrm{b}}\right), 26.7\left(\mathrm{C}_{\mathrm{c}}+\mathrm{C}_{\mathrm{d}}\right)$; HRMS (ESI) calcd for $\mathrm{C}_{9} \mathrm{H}_{16} \mathrm{O}_{3} \mathrm{Na}^{+}[\mathrm{M}+\mathrm{Na}]^{+}$195.0992, found 195.0987; IR $\nu_{\max }$ (film) $3460,2928,2863,1106,1032,928,890 \mathrm{~cm}^{-1}$. The data presented are consistent with literature precedent. ${ }^{39}$

tert-Butyl 4-(Hydroxymethyl)piperidine-1-carboxylate (4f). General procedure B was followed using tert-butyl 3-methoxy-3-methyl-4oxa-1,2,8-triazaspiro[4.5] dec-1-ene-8-carboxylate $(57 \mathrm{mg}, 0.2 \mathrm{mmol}$, 1.0 equiv), formaldehyde $\left(0.16 \mathrm{~mL}, 37 \mathrm{wt} \%\right.$ in $\mathrm{H}_{2} \mathrm{O}, 2.0 \mathrm{mmol}, 10$ equiv), and sodium borohydride ( $76 \mathrm{mg}, 2.0 \mathrm{mmol}, 10$ equiv). The crude mixture was purified via flash column chromatography (10$50 \% \mathrm{EtOAc}$ in petroleum ether) to give the titled product as a colorless oil (23 mg, 53\%): ${ }^{1} \mathrm{H} \mathrm{NMR}\left(600 \mathrm{MHz}, \mathrm{CDCl}_{3}\right) \delta 4.12$ (br s, $\left.2 \mathrm{H}, \mathrm{NCH}_{\mathrm{c}}+\mathrm{NCH}_{\mathrm{d}}\right), 3.56-3.44\left(\mathrm{~m}, 2 \mathrm{H}, \mathrm{HOCH}_{2} \mathrm{CH}\right), 2.69$ (br s, $\left.2 \mathrm{H}, \mathrm{NCH}_{\mathrm{c}}{ }^{\prime}+\mathrm{NCH}_{\mathrm{d}}{ }^{\prime}\right), 1.75-1.68\left(\mathrm{~m}, 2 \mathrm{H}, \mathrm{CH}_{\mathrm{a}}+\mathrm{NCH}_{\mathrm{b}}\right), 1.66-1.59$ $\left(\mathrm{m}, 1 \mathrm{H}, \mathrm{HOCH}_{2} \mathrm{CH}\right), 1.45\left(\mathrm{~s}, 9 \mathrm{H}, \mathrm{C}\left(\mathrm{CH}_{3}\right)_{3}\right), 1.20-1.05(\mathrm{~m}, 2 \mathrm{H}$, $\left.\mathrm{CH}_{\mathrm{a}}{ }^{\prime}+\mathrm{CH}_{\mathrm{b}}{ }^{\prime}\right) ;{ }^{13} \mathrm{C}\left\{{ }^{1} \mathrm{H}\right\}$ NMR $\left(151 \mathrm{MHz}, \mathrm{CDCl}_{3}\right) \delta 155.0(\mathrm{NCOO})$, $79.5\left(\mathrm{C}\left(\mathrm{CH}_{3}\right)_{3}\right), 67.8\left(\mathrm{HOCH}_{2}\right), 43.8\left(\mathrm{br}, \mathrm{C}_{\mathrm{c}}+\mathrm{C}_{\mathrm{d}}\right), 39.0$ $\left(\mathrm{HOCH}_{2} \mathrm{CH}\right), 28.7\left(\mathrm{br}, \mathrm{C}_{\mathrm{a}}+\mathrm{C}_{\mathrm{b}}\right), 28.6\left(\mathrm{C}\left(\mathrm{CH}_{3}\right)_{3}\right)$; HRMS (ESI) calcd for $\mathrm{C}_{11} \mathrm{H}_{22} \mathrm{O}_{3} \mathrm{~N}^{+}[\mathrm{M}+\mathrm{H}]^{+} 216.1594$, found 216.1591; IR $\nu_{\max }$ (film) 3455, 2974, 2924, 2857, 2355, 1693, 1669, 1424, 1366, 1313, $1274,1247,1168,1087,1039,962,864,769 \mathrm{~cm}^{-1}$. The data presented are consistent with literature precedent. ${ }^{40}$

(Adamantan-2-yl)methanol (4h). General procedure B was followed using $5^{\prime}$-methoxy-5' ${ }^{\prime}$-methyl-5' $H$-spiro[adamantane-2, $2^{\prime}$ $[1,3,4]$ oxadiazole $]$ ( $94 \mathrm{mg}, 0.4 \mathrm{mmol}, 1.0$ equiv), formaldehyde (0.3 $\mathrm{mL}, 37 \mathrm{wt} \%$ in $\mathrm{H}_{2} \mathrm{O}, 4 \mathrm{mmol}, 10$ equiv), and sodium borohydride (153 mg, $2.0 \mathrm{mmol}, 10$ equiv). The crude mixture was purified via flash column chromatography (10-40\% EtOAc in petroleum ether) to give the titled product as a transparent oil $(50 \mathrm{mg}, 75 \%):{ }^{1} \mathrm{H}$ NMR $\left(600 \mathrm{MHz}, \mathrm{CDCl}_{3}\right) \delta 3.74\left(\mathrm{~d}, \mathrm{~J}=7.1 \mathrm{~Hz}, 2 \mathrm{H}, \mathrm{HOCH}_{2}\right), 1.94-1.90$ $\left(\mathrm{m}, 1 \mathrm{H}, \mathrm{HOCH}_{2} \mathrm{CH}\right), 1.89-1.84\left(\mathrm{~m}, 4 \mathrm{H}, \mathrm{CH}_{\mathrm{b}}\right), 1.83-1.79(\mathrm{~m}, 3 \mathrm{H}$, $\left.\mathrm{CH}_{\mathrm{b}}{ }^{\prime}+\mathrm{CH}_{\mathrm{a}}\right), 1.79-1.77\left(\mathrm{~m}, 1 \mathrm{H}, \mathrm{CH}_{\mathrm{a}}{ }^{\prime}\right), 1.75-1.72\left(\mathrm{~m}, 2 \mathrm{H}, \mathrm{CH}_{\mathrm{b}}{ }^{\prime \prime}\right)$, 1.57 (br s, $1 \mathrm{H}, \mathrm{CH}_{\mathrm{c}}$ ), 1.55 (br s, $\left.3 \mathrm{H}, \mathrm{CH}_{\mathrm{c}}{ }^{\prime}+\mathrm{CH}_{\mathrm{d}}\right), 1.25(\mathrm{br} \mathrm{s}, 1 \mathrm{H}$, $\mathrm{HO}) ;{ }^{13} \mathrm{C}\left\{{ }^{1} \mathrm{H}\right\}$ NMR $\left(151 \mathrm{MHz}, \mathrm{CDCl}_{3}\right) \delta 65.3\left(\mathrm{HOCH}_{2}\right), 47.3$ $\left(\mathrm{HOCH}_{2} \mathrm{CH}\right), 39.1\left(C_{\mathrm{b}}\right), 38.2\left(C_{\mathrm{d}}\right), 31.9\left(C_{\mathrm{b}}{ }^{\prime}\right), 29.2\left(C_{\mathrm{a}}\right), 28.4\left(C_{\mathrm{c}}\right)$, $27.9\left(C_{\mathrm{c}}{ }^{\prime}\right)$; HRMS $(\mathrm{ESI})$ calcd for $\mathrm{C}_{11} \mathrm{H}_{18} \mathrm{ONa}^{+}[\mathrm{M}+\mathrm{Na}]^{+}$189.1250, found 189.1247; IR $\nu_{\text {max }}$ (film) 3260, 2861, 2849, 1466, 1452, 1066, 1033, 1007, $971 \mathrm{~cm}^{-1}$. The data presented are consistent with literature precedent. ${ }^{41}$

4-(6-Methoxynaphthalen-2-yl)-2-methylbutan-1-ol (4j). General procedure B was followed using 2-methoxy-5-(2-(6-methoxynaphthalen-2-yl)ethyl)-2,5-dimethyl-2,5-dihydro-1,3,4-oxadiazole (98 mg, $0.4 \mathrm{mmol}, 1.0$ equiv), formaldehyde $\left(0.3 \mathrm{~mL}, 37\right.$ wt $\%$ in $\mathrm{H}_{2} \mathrm{O}, 4$ mmol, 10 equiv), and sodium borohydride ( $153 \mathrm{mg}, 2.0 \mathrm{mmol}, 10$ equiv). The crude mixture was purified via flash column chromatography (10-40\% EtOAc in petroleum ether) to give the titled product as a yellow oil $(73 \mathrm{mg}, 75 \%):{ }^{1} \mathrm{H}$ NMR $(600 \mathrm{MHz}$, $\left.\mathrm{CDCl}_{3}\right) \delta 7.66\left(\mathrm{~d}, \mathrm{~J}=8.4 \mathrm{~Hz}, 2 \mathrm{H}, H_{\mathrm{Ar}}\right), 7.55\left(\mathrm{~s}, 1 \mathrm{H}, H_{\mathrm{Ar}}\right), 7.33(\mathrm{dd}, \mathrm{J}$ $\left.=8.3,1.8 \mathrm{~Hz}, 1 \mathrm{H}, H_{\mathrm{Ar}}\right), 7.14-7.09\left(\mathrm{~m}, 2 \mathrm{H}, H_{\mathrm{Ar}}\right), 3.91\left(\mathrm{~s}, 3 \mathrm{H}, \mathrm{OCH}_{3}\right)$, $3.56\left(\right.$ br s, $\left.1 \mathrm{H}, \mathrm{HOCH}_{\mathrm{c}}\right), 3.54-3.51\left(\mathrm{~m}, 1 \mathrm{H}, \mathrm{HOCH}_{\mathrm{c}}{ }^{\prime}\right), 2.89-2.79$ $\left(\mathrm{m}, 1 \mathrm{H}, \mathrm{ArCH}_{\mathrm{a}}\right), 2.78-2.68\left(\mathrm{~m}, 1 \mathrm{H}, \mathrm{ArCH}_{\mathrm{a}}{ }^{\prime}\right), 1.91-1.80(\mathrm{~m}, 1 \mathrm{H}$, $\left.\mathrm{CH}_{\mathrm{b}}\right), 1.76-1.66\left(\mathrm{~m}, 1 \mathrm{H}, \mathrm{CH}_{\mathrm{b}}{ }^{\prime}\right), 1.61-1.50\left(\mathrm{~m}, 2 \mathrm{H}, \mathrm{HOCH}_{2} \mathrm{CH}+\right.$ $\mathrm{OH}), 1.02\left(\mathrm{~d}, \mathrm{~J}=6.7 \mathrm{~Hz}, 3 \mathrm{H}, \mathrm{CHCH}_{3}\right) ;{ }^{13} \mathrm{C}\left\{{ }^{1} \mathrm{H}\right\}$ NMR $(151 \mathrm{MHz}$, $\left.\mathrm{CDCl}_{3}\right) \delta 157.3\left(C_{\mathrm{Ar}}\right), 137.9\left(C_{\mathrm{Ar}}\right), 133.1\left(C_{\mathrm{Ar}}\right), 129.2\left(C_{\mathrm{Ar}}\right), 129.0$ $\left(C_{\mathrm{Ar}} \mathrm{H}\right), 127.9\left(C_{\mathrm{Ar}} \mathrm{H}\right), 126.9\left(C_{\mathrm{Ar}} \mathrm{H}\right), 126.3\left(C_{\mathrm{Ar}} \mathrm{H}\right), 118.6\left(C_{\mathrm{Ar}} \mathrm{H}\right)$, $105.6\left(C_{\mathrm{Ar}} \mathrm{H}\right), 68.2\left(C_{\mathrm{c}}\right), 55.3\left(\mathrm{OCH}_{3}\right), 35.3\left(C_{\mathrm{a}}\right), 34.9\left(C_{\mathrm{b}}\right), 33.2$ $\left(\mathrm{HOCH}_{2} \mathrm{CH}\right), 16.5\left(\mathrm{CHCH}_{3}\right)$; HRMS (ESI) calcd for $\mathrm{C}_{16} \mathrm{H}_{20} \mathrm{O}_{2}{ }^{+}[\mathrm{M}$ $+\mathrm{H}]^{+}$244.1467, found 244.1463; IR $\nu_{\max }$ (film) 3342, 2961, 2926, 2850, 1634, 1604, 1484, 1462, 1391, 1263, $1228 \mathrm{~cm}^{-1}$.

2-Methylpentane-1,5-diol (4k). General procedure B was followed using 3-(5-methoxy-2,5-dimethyl-2,5-dihydro-1,3,4-oxadiazol-2-yl)propan-1-ol (37 mg, $0.2 \mathrm{mmol}, 1.0$ equiv), formaldehyde $(0.16 \mathrm{~mL}$, 37 wt \% in $\mathrm{H}_{2} \mathrm{O}, 2.0 \mathrm{mmol}, 10$ equiv), and sodium borohydride (76 $\mathrm{mg}, 2.0 \mathrm{mmol}, 10$ equiv). The crude mixture was purified via flash 
column chromatography ( $2 \% \mathrm{MeOH}$ in dichloromethane) to give the titled product as a colorless oil $(16 \mathrm{mg}, 66 \%):{ }^{1} \mathrm{H}$ NMR $(600 \mathrm{MHz}$, $\left.\mathrm{CDCl}_{3}\right) \delta 3.60\left(\mathrm{t}, J=6.1 \mathrm{~Hz}, 2 \mathrm{H}, \mathrm{HOCH}_{2} \mathrm{CH}_{2}\right), 3.48-3.36(\mathrm{~m}, 2 \mathrm{H}$, $\mathrm{HOCH}_{2} \mathrm{CH}$ ), 3.16 (br s, $\left.2 \mathrm{H}, \mathrm{OH}\right), 1.66-1.56\left(\mathrm{~m}, 2 \mathrm{H}, \mathrm{HOCH}_{2} \mathrm{CH}_{2}\right)$, 1.54-1.45 (m, 2H, $\left.\mathrm{CH}_{2} \mathrm{CH}_{2} \mathrm{CHCH}_{3}\right), 1.16-1.06\left(\mathrm{~m}, 1 \mathrm{H}, \mathrm{CHCH}_{3}\right)$, $0.88\left(\mathrm{~d}, J=6.7 \mathrm{~Hz}, 3 \mathrm{H}, \mathrm{CH}_{3}\right) ;{ }^{13} \mathrm{C}\left\{{ }^{1} \mathrm{H}\right\} \mathrm{NMR}\left(151 \mathrm{MHz}, \mathrm{CDCl}_{3}\right) \delta$ $67.8\left(\mathrm{HOCH}_{2} \mathrm{CH}_{2}\right), 62.8\left(\mathrm{HOCH}_{2} \mathrm{CH}\right), 35.4\left(\mathrm{HOCH}_{2} \mathrm{CH}_{2}\right), 29.7$ $\left(\mathrm{CH}_{2} \mathrm{CH}_{2} \mathrm{CHCH}_{3}\right), 29.1\left(\mathrm{CHCH}_{3}\right), 16.7\left(\mathrm{CH}_{3}\right)$; HRMS (ESI) calcd for $\mathrm{C}_{6} \mathrm{H}_{15} \mathrm{O}_{2}^{+}[\mathrm{M}+\mathrm{H}]^{+} 119.1067$, found 119.1066; IR $\nu_{\max }$ (film) 3291, 2932, 2869, 1652, 1455, 1418, 1377, 1104, 1038, 940, 897, 731 $\mathrm{cm}^{-1}$. The data presented are consistent with literature precedent. ${ }^{42}$

2-Methyl-3-(pyridin-4-yl)propan-1-ol (4I). General procedure B was followed using 2-methoxy-2,5-dimethyl-5-(pyridin-4-ylmethyl)2,5-dihydro-1,3,4-oxadiazole ( $88 \mathrm{mg}, 0.4 \mathrm{mmol}, 1.0$ equiv), formaldehyde ( $0.3 \mathrm{~mL}, 37 \mathrm{wt} \%$ in $\mathrm{H}_{2} \mathrm{O}, 4 \mathrm{mmol}, 10$ equiv), and sodium borohydride ( $153 \mathrm{mg}, 2.0 \mathrm{mmol}, 10$ equiv). The crude mixture was purified via flash column chromatography $(30-70 \%$ EtOAc in petroleum ether) to give the titled product as a transparent oil (30 mg, 50\%): ${ }^{1} \mathrm{H}$ NMR $\left(600 \mathrm{MHz}, \mathrm{CDCl}_{3}\right) \delta 8.51$ (br s, $\left.2 \mathrm{H}, \mathrm{H}_{\mathrm{Ar}}\right), 7.12$ $\left(\mathrm{d}, J=4.9 \mathrm{~Hz}, 2 \mathrm{H}, H_{\mathrm{Ar}}\right), 3.51\left(\mathrm{dd}, J=5.9,1.0 \mathrm{~Hz}, 2 \mathrm{H}, \mathrm{HOCH}_{2}\right), 2.81$ (dd, $\left.J=13.4,6.0 \mathrm{~Hz}, 1 \mathrm{H}, \mathrm{CH}_{\mathrm{a}}\right), 2.40\left(\mathrm{dd}, J=13.4,8.4 \mathrm{~Hz}, 1 \mathrm{H}, \mathrm{CH}_{\mathrm{a}}{ }^{\prime}\right)$, 2.03-1.93 (m, 1H, $\left.\mathrm{HOCH}_{2} \mathrm{CH}\right), 1.75($ br s, $1 \mathrm{H}, \mathrm{OH}), 0.91(\mathrm{~d}, J=$ $\left.6.8 \mathrm{~Hz}, 3 \mathrm{H}, \mathrm{CHCH}_{3}\right) ;{ }^{13} \mathrm{C}\left\{{ }^{1} \mathrm{H}\right\}$ NMR $\left(151 \mathrm{MHz}, \mathrm{CDCl}_{3}\right) \delta 150.2$ $\left(C_{\text {pyridine }}\right), 149.6\left(C_{\text {pyridine }} \mathrm{H}\right), 124.8\left(C_{\text {pyridine }} \mathrm{H}\right), 67.2\left(\mathrm{HOCH}_{2}\right), 39.0$ $\left(C_{\mathrm{a}}\right), 37.2\left(\mathrm{HOCH}_{2} \mathrm{CH}\right), 16.4\left(\mathrm{CHCH}_{3}\right)$; HRMS (ESI) calcd for $\mathrm{C}_{9} \mathrm{H}_{14} \mathrm{NO}^{+}[\mathrm{M}+\mathrm{H}]^{+}$152.1071, found 152.1075; IR $\nu_{\max }$ (film) 3353, $2924,2348,2185,1605,1043 \mathrm{~cm}^{-1}$. The data presented are consistent with literature precedent. ${ }^{43}$

3-(Furan-2-yl)-2-methylpropan-1-ol (4m). General procedure B was followed using 2-(furan-2-ylmethyl)-5-methoxy-2,5-dimethyl-2,5dihydro-1,3,4-oxadiazole ( $84 \mathrm{mg}, 0.4 \mathrm{mmol}, 1.0$ equiv), formaldehyde (0.3 mL, 37 wt \% in $\mathrm{H}_{2} \mathrm{O}, 4 \mathrm{mmol}, 10$ equiv), and sodium borohydride ( $152 \mathrm{mg}, 4.0 \mathrm{mmol}, 10$ equiv). The crude mixture was purified via flash column chromatography $(10-40 \%$ EtOAc in petroleum ether) to give the titled product as a transparent oil (34 mg, 60\%): ${ }^{1} \mathrm{H}$ NMR $\left(600 \mathrm{MHz}, \mathrm{CDCl}_{3}\right) \delta 7.31(\mathrm{dd}, J=1.9,0.9 \mathrm{~Hz}$, $\left.1 \mathrm{H}, H_{\text {Furan }}\right), 6.29\left(\mathrm{dd}, J=3.2,1.9 \mathrm{~Hz}, 1 \mathrm{H}, H_{\text {Furan }}\right), 6.02(\mathrm{dd}, J=3.2$, $\left.0.9 \mathrm{~Hz}, 1 \mathrm{H}, H_{\text {Furan }}\right), 3.50\left(\mathrm{~d}, J=6.0 \mathrm{~Hz}, 2 \mathrm{H}, \mathrm{HOCH}_{2}\right), 2.73(\mathrm{dd}, J=$ $\left.14.9,6.4 \mathrm{~Hz}, 1 \mathrm{H}, \mathrm{CH}_{\mathrm{a}}\right), 2.54\left(\mathrm{dd}, J=14.9,7.4 \mathrm{~Hz}, 1 \mathrm{H}, \mathrm{CH}_{\mathrm{a}}{ }^{\prime}\right), 2.03$ $\left(\mathrm{dq}, J=13.2,6.4 \mathrm{~Hz}, 1 \mathrm{H}, \mathrm{HOCH}_{2} \mathrm{CH}\right), 1.46(\mathrm{br} \mathrm{s}, 1 \mathrm{H}, \mathrm{OH}), 0.94(\mathrm{~d}$, $\left.J=6.8 \mathrm{~Hz}, 3 \mathrm{H}, \mathrm{CHCH}_{3}\right) ;{ }^{13} \mathrm{C}\left\{{ }^{1} \mathrm{H}\right\} \operatorname{NMR}\left(151 \mathrm{MHz}, \mathrm{CDCl}_{3}\right) \delta 154.7$ $\left(C_{\text {Furan }}\right), 141.2\left(C_{\text {Furan }} \mathrm{H}\right), 110.3\left(C_{\text {Furan }} \mathrm{H}\right), 106.3\left(C_{\text {Furan }} \mathrm{H}\right), 67.6$ $\left(\mathrm{HOCH}_{2}\right), 35.4\left(\mathrm{C}_{\mathrm{a}}\right), 31.5\left(\mathrm{HOCH}_{2} \mathrm{CH}\right), 16.5\left(\mathrm{CHCH}_{3}\right) ; \mathrm{HRMS}$ (ESI) calcd for $\mathrm{C}_{8} \mathrm{H}_{11} \mathrm{O}_{2}{ }^{-}[\mathrm{M}-\mathrm{H}]^{-}$139.0754, found 139.0753; IR $\nu_{\max }($ film) 3342, 2919,1595, 1507, 1460, 1381, 1146, 1033, 927 $\mathrm{cm}^{-1}$. The data presented are consistent with literature precedent. ${ }^{44}$

2-Methylhex-5-en-1-ol (4n). General procedure B was followed using 2-(but-3-en-1-yl)-5-methoxy-2,5-dimethyl-2,5-dihydro-1,3,4-oxadiazole ( $37 \mathrm{mg}, 0.2 \mathrm{mmol}, 1.0$ equiv), formaldehyde $(0.16 \mathrm{~mL}, 37 \mathrm{wt}$ $\%$ in $\mathrm{H}_{2} \mathrm{O}, 2.0 \mathrm{mmol}, 10$ equiv), and sodium borohydride ( $76 \mathrm{mg}, 2.0$ mmol, 10 equiv). An $88 \%$ NMR yield was calculated using 1,3,5trimethoxybenzene ( $11 \mathrm{mg}, 0.066 \mathrm{mmol}, 0.33$ equiv) as an internal standard. The crude mixture was purified via flash column chromatography (0-40\% EtOAc in petroleum ether) to give the titled product as a colorless oil $(16 \mathrm{mg}, 69 \%):{ }^{1} \mathrm{H}$ NMR $(600 \mathrm{MHz}$, $\left.\mathrm{CDCl}_{3}\right) \delta 5.81\left(\mathrm{ddt}, J=17.0,10.2,6.6 \mathrm{~Hz}, 1 \mathrm{H}, \mathrm{CH}_{2}=\mathrm{CH}\right), 5.02(\mathrm{dd}$, $\left.J=17.0,1.9 \mathrm{~Hz}, 1 \mathrm{H}, H_{\mathrm{b}}\right), 4.95\left(\mathrm{dd}, J=10.2,1.9 \mathrm{~Hz}, 1 \mathrm{H}, H_{\mathrm{a}}\right), 3.48$ (ddd, $\left.J=45.3,10.5,6.1 \mathrm{~Hz}, 2 \mathrm{H}, \mathrm{CHCH}_{2} \mathrm{OH}\right), 2.18-2.09(\mathrm{~m}, 1 \mathrm{H}$, $\left.\mathrm{CH}_{2}=\mathrm{CHCH}_{\mathrm{c}}\right), 2.09-1.99\left(\mathrm{~m}, 1 \mathrm{H}, \mathrm{CH}_{2}=\mathrm{CHCH}_{\mathrm{c}}{ }^{\prime}\right), 1.70-1.60(\mathrm{~m}$, $\left.1 \mathrm{H}, \mathrm{CH}_{2} \mathrm{CH}_{\mathrm{d}} \mathrm{CHCH}_{3}\right), 1.58-1.47\left(\mathrm{~m}, 1 \mathrm{H}, \mathrm{CH}_{2} \mathrm{CH}_{\mathrm{d}}{ }^{\prime} \mathrm{CHCH}_{3}\right), 1.33$ (br s, $1 \mathrm{H}, \mathrm{OH}), 1.28-1.16\left(\mathrm{~m}, 1 \mathrm{H}, \mathrm{CHCH}_{3}\right), 0.93(\mathrm{~d}, J=6.7 \mathrm{~Hz}, 3 \mathrm{H}$, $\left.\mathrm{CH}_{3}\right) ;{ }^{13} \mathrm{C}\left\{{ }^{1} \mathrm{H}\right\}$ NMR $\left(151 \mathrm{MHz}, \mathrm{CDCl}_{3}\right) \delta 139.0\left(\mathrm{CH}_{2}=\mathrm{CH}\right), 114.6$ $\left(\mathrm{CH}_{2}=\mathrm{CH}\right), 68.3\left(\mathrm{CHCH}_{2} \mathrm{OH}\right), 35.4\left(\mathrm{C}_{\mathrm{c}}\right), 32.4\left(\mathrm{C}_{\mathrm{d}}\right), 31.3$ $\left(\mathrm{CHCH}_{3}\right), 16.6\left(\mathrm{CH}_{3}\right)$; LRMS (ESI, $\left.m / z\right) 113.5\left([\mathrm{M}+\mathrm{H}]^{+}, 100\right)$; IR $\nu_{\max }$ (film) 3436, 2969, 2355, 2316, 1448, 1329, $1046 \mathrm{~cm}^{-1}$. The data presented are consistent with literature precedent. ${ }^{45}$

Synthesis of Benzimidazoles. General Procedure $C$ for the Synthesis of Benzimidazoles. The procedure was a modification of the literature procedure from Jiao et al. ${ }^{26}$
The reaction slug from general procedure A was collected into a round-bottom flask containing a biphasic solution of brine and toluene with stirring. Upon resting, the toluene phase was syringed out and injected into another open round-bottom flask charged with freshly activated $4 \AA$ molecular sieves. The mixture was stirred for another $1 \mathrm{~min}$ before $o$-phenylenediamine ( 1.5 equiv) was added. The reaction mixture was then bubbled with one $\mathrm{O}_{2}$ balloon and stirred at room temperature $\left(30{ }^{\circ} \mathrm{C}\right)$ for $12 \mathrm{~h}$. Molecular sieves were filtered over filter paper, and the filtrate was concentrated under vacuum before being purified via flash chromatography with appropriate eluent combinations to afford the final benzimidazole derivatives.

2-(1,2,3,4-Tetrahydronaphthalen-2-yl)-1H-benzimidazole (5a). General procedure $C$ was followed using $5^{\prime}$-methoxy-5'-methyl-3,4dihydro- $1 H, 5^{\prime} H$-spiro[naphthalene-2,2'-[1,3,4] oxadiazole] (46 mg, $0.2 \mathrm{mmol}, 1.0$ equiv), formaldehyde $\left(0.15 \mathrm{~mL}, 37\right.$ wt $\%$ in $\mathrm{H}_{2} \mathrm{O}, 2$ mmol, 10 equiv), and o-phenylenediamine $(32 \mathrm{mg}, 0.3 \mathrm{mmol}, 1.5$ equiv). The crude mixture was purified via flash column chromatography (10-40\% EtOAc in petroleum ether) to give the titled product as a white solid $(36 \mathrm{mg}, 72 \%):{ }^{1} \mathrm{H}$ NMR $(600 \mathrm{MHz}$, $\left.\mathrm{CDCl}_{3}\right) \delta 9.06($ br s, $1 \mathrm{H}, \mathrm{NH}), 7.76\left(\right.$ br s, $\left.1 \mathrm{H}, \mathrm{H}_{\mathrm{Ar}}\right), 7.40($ br s, $1 \mathrm{H}$, $\left.H_{\mathrm{Ar}}\right), 7.24\left(\mathrm{~d}, J=5.5 \mathrm{~Hz}, 2 \mathrm{H}, H_{\mathrm{Ar}}\right), 7.29-7.13\left(\mathrm{~m}, 4 \mathrm{H}, H_{\mathrm{Ar}}\right), 3.41$ (tdd, $J=10.3,5.5,3.1 \mathrm{~Hz}, 1 \mathrm{H}, \mathrm{CCH}), 3.35-3.20(\mathrm{~m}, 2 \mathrm{H}$, $\mathrm{ArCH}_{2} \mathrm{CH}$ ), 2.97 (qp, $J=10.3,5.5 \mathrm{~Hz}, 2 \mathrm{H}, \mathrm{ArCH}_{2} \mathrm{CH}_{2}$ ), 2.48$2.37\left(\mathrm{~m}, 1 \mathrm{H}, \mathrm{CHCH}_{\mathrm{a}}^{\prime}\right), 2.14(\mathrm{dtd}, J=13.0,10.3,6.2 \mathrm{~Hz}, 1 \mathrm{H}$, $\left.\mathrm{CHCH}_{\mathrm{c}}{ }^{\prime}\right) ;{ }^{13} \mathrm{C}\left\{{ }^{1} \mathrm{H}\right\}$ NMR $\left(151 \mathrm{MHz}\right.$, methanol- $\left.d_{4}\right) \delta 159.8\left(C_{\mathrm{Ar}}\right)$, $136.8\left(C_{\mathrm{Ar}}\right), 136.1\left(C_{\mathrm{Ar}}\right), 130.0\left(C_{\mathrm{Ar}} \mathrm{H}\right), 129.9\left(C_{\mathrm{Ar}} \mathrm{H}\right), 127.1\left(C_{\mathrm{Ar}} \mathrm{H}\right)$, $126.9\left(C_{\mathrm{Ar}} \mathrm{H}\right), 123.3\left(C_{\mathrm{Ar}} \mathrm{H}\right), 115.4\left(\mathrm{br}, \mathrm{C}_{\mathrm{Ar}} \mathrm{H}\right), 36.7(\mathrm{CCH}), 35.4$ $\left(\mathrm{ArCH}_{2} \mathrm{CH}\right), 30.0\left(\mathrm{ArCH}_{2} \mathrm{CH}_{2}\right), 29.6\left(\mathrm{C}_{a}\right)$; One aromatic carbon is not seen in the ${ }^{13} \mathrm{C}\left\{{ }^{1} \mathrm{H}\right\}$ NMR spectrum due to peak broadening; HRMS (ESI) calcd for $\mathrm{C}_{17} \mathrm{H}_{17} \mathrm{~N}_{2}^{+}[\mathrm{M}+\mathrm{H}]^{+}$249.1387, found 249.1392; IR $\nu_{\max }$ (film) 2921, 1423, 1275, 1009, 993, 932, $743 \mathrm{~cm}^{-1}$. Mp: $239-241{ }^{\circ} \mathrm{C}$.

2-(Tetrahydro-2H-pyran-4-yl)-1H-benzimidazole (5b). General procedure $\mathrm{C}$ was followed using 3-methoxy-3-methyl-4,8-dioxa-1,2diazaspiro[4.5] dec-1-ene (37 mg, $0.2 \mathrm{mmol}, 1.0$ equiv), formaldehyde ( $0.15 \mathrm{~mL}, 37$ wt $\%$ in $\mathrm{H}_{2} \mathrm{O}, 2 \mathrm{mmol}, 10$ equiv), and $o$ phenylenediamine ( $32 \mathrm{mg}, 0.3 \mathrm{mmol}, 1.5$ equiv). The crude mixture was purified via flash column chromatography $(10-40 \%$ EtOAc in petroleum ether) to give the titled product as a transparent oil (31 $\mathrm{mg}, 76 \%):{ }^{1} \mathrm{H}$ NMR $\left(600 \mathrm{MHz}\right.$, methanol- $\left.d_{4}\right) \delta 7.51(\mathrm{dd}, J=6.1,3.2$ $\left.\mathrm{Hz}, 2 \mathrm{H}, H_{\mathrm{Ar}}\right), 7.19\left(\mathrm{dd}, J=6.1,3.2 \mathrm{~Hz}, 2 \mathrm{H}, H_{\mathrm{Ar}}\right), 4.06(\mathrm{dt}, J=11.5$, $\left.3.3 \mathrm{~Hz}, 2 \mathrm{H}, \mathrm{OCH}_{\mathrm{c}}+\mathrm{OCH}_{\mathrm{d}}\right), 3.65-3.53\left(\mathrm{~m}, 2 \mathrm{H}, \mathrm{OCH}_{\mathrm{c}}{ }^{\prime}+\mathrm{OCH}_{\mathrm{d}}{ }^{\prime}\right)$, 3.24-3.15 (m, $1 \mathrm{H}, \mathrm{CCH}), 2.04-1.92\left(\mathrm{~m}, 4 \mathrm{H}, \mathrm{CH}_{\mathrm{a}}+\mathrm{OCH}_{\mathrm{b}}\right)$; ${ }^{13} \mathrm{C}\{\mathrm{H}\}$ NMR $\left(151 \mathrm{MHz}\right.$, Methanol- $\left.d_{4}\right) \delta 159.0\left(C_{\mathrm{Ar}}\right), 123.3\left(C_{A r} \mathrm{H}\right)$, $115.2\left(\mathrm{br}, C_{\mathrm{Ar}} \mathrm{H}\right), 68.6\left(C_{c}+C_{d}\right), 36.8(\mathrm{CCH}), 32.4\left(C_{\mathrm{a}}+C_{\mathrm{b}}\right)$; one aromatic carbon is not seen in the ${ }^{13} \mathrm{C}\left\{{ }^{1} \mathrm{H}\right\}$ NMR spectrum due to peak broadening; HRMS (ESI) calcd for $\mathrm{C}_{12} \mathrm{H}_{15} \mathrm{~N}_{2} \mathrm{O}^{+}[\mathrm{M}+\mathrm{H}]^{+}$ 203.1181, found 203.1184; IR $\nu_{\max }$ (film) 2958, 2922, 2852, 1457, 1427, 1128, $739 \mathrm{~cm}^{-1}$. Mp: $225-227^{\circ} \mathrm{C}$.

2-(Tetrahydro-2H-thiopyran-4-yl)-1H-benzimidazole (5c). General procedure $\mathrm{C}$ was followed using 3-methoxy-3-methyl-4-oxa-8thia-1,2-diazaspiro[4.5] dec-1-ene (40 mg, $0.2 \mathrm{mmol}, 1.0$ equiv), formaldehyde ( $0.16 \mathrm{~mL}, 37 \mathrm{wt} \%$ in $\mathrm{H}_{2} \mathrm{O}, 2.0 \mathrm{mmol}, 10$ equiv), and $o$ phenylenediamine ( $32 \mathrm{mg}, 0.3 \mathrm{mmol}, 1.5$ equiv). The crude mixture was purified via flash column chromatography $(10-40 \%$ EtOAc in petroleum ether) to give the titled product as a white solid $(24 \mathrm{mg}$, 55\%): ${ }^{1} \mathrm{H}$ NMR (600 MHz, methanol- $\left.d_{4}\right) \delta 7.51$ (br s, $\left.2 \mathrm{H}, H_{\mathrm{Ar}}\right), 7.20$ $\left(\mathrm{dd}, J=6.1,3.1 \mathrm{~Hz}, 2 \mathrm{H}, H_{\mathrm{Ar}}\right), 4.59(\mathrm{~s}, 1 \mathrm{H}, \mathrm{NH}), 3.00$ (ddd, $J=12.0$, 8.7, $3.3 \mathrm{~Hz}, 1 \mathrm{H}, \mathrm{CCH}), 2.92-2.85\left(\mathrm{~m}, 2 \mathrm{H}, \mathrm{SCH}_{\mathrm{c}}+\mathrm{SCH}_{\mathrm{d}}\right), 2.73(\mathrm{~d}, J$ $\left.=14.0 \mathrm{~Hz}, 2 \mathrm{H}, \mathrm{SCH}_{\mathrm{c}}{ }^{\prime}+\mathrm{SCH}_{\mathrm{b}}{ }^{\prime}\right), 2.37\left(\mathrm{dd}, J=13.6,3.1 \mathrm{~Hz}, 2 \mathrm{H}, \mathrm{CH}_{\mathrm{a}}\right.$ $\left.+\mathrm{CH}_{\mathrm{b}}\right), 2.06\left(\mathrm{qd}, J=12.5,3.2 \mathrm{~Hz}, 2 \mathrm{H}, \mathrm{CH}_{\mathrm{a}}+\mathrm{CH}_{\mathrm{b}}\right) ;{ }^{13} \mathrm{C}\left\{{ }^{1} \mathrm{H}\right\} \mathrm{NMR}$ $\left(151 \mathrm{MHz}\right.$, methanol- $\left.d_{4}\right) \delta 159.7\left(C_{\mathrm{Ar}}\right), 123.3\left(\mathrm{br}, C_{\mathrm{Ar}} \mathrm{H}\right), 39.5$ $(\mathrm{CCH}), 33.8\left(C_{\mathrm{a}}+C_{\mathrm{b}}\right), 29.2\left(C_{\mathrm{c}}+C_{\mathrm{d}}\right)$; two aromatic carbons are not seen in the ${ }^{13} \mathrm{C}\left\{{ }^{1} \mathrm{H}\right\}$ NMR spectrum due to peak broadening; HRMS (ESI) calcd for $\mathrm{C}_{12} \mathrm{H}_{15} \mathrm{~N}_{2} \mathrm{~S}^{+}[\mathrm{M}+\mathrm{H}]^{+}$219.0956, found 219.0955; IR $\nu_{\max }$ (film) 3394, 2924, 1709, 1432, 1274, 1047, 951, $744 \mathrm{~cm}^{-1}$; mp $220-222{ }^{\circ} \mathrm{C}$.

2-(Tetrahydrothiophene-3-yl)-1H-benzimidazole (5d). General procedure $\mathrm{C}$ was followed using 3-methoxy-3-methyl-4-oxa-7-thia1,2-diazaspiro[4.4]non-1-ene ( $38 \mathrm{mg}, 0.2 \mathrm{mmol}, 1.0$ equiv), form- 
aldehyde ( $0.15 \mathrm{~mL}, 37$ wt $\%$ in $\mathrm{H}_{2} \mathrm{O}, 2 \mathrm{mmol}, 10$ equiv), and $o$ phenylenediamine ( $32 \mathrm{mg}, 0.3 \mathrm{mmol}, 1.5$ equiv). The crude mixture was purified via flash column chromatography $(10-40 \%$ EtOAc in petroleum ether) to give the titled product as a white solid $(29 \mathrm{mg}$, $72 \%):{ }^{1} \mathrm{H}$ NMR $\left(600 \mathrm{MHz}\right.$, methanol- $\left.d_{4}\right) \delta 7.55-7.38\left(\mathrm{~m}, 2 \mathrm{H}, H_{\mathrm{Ar}}\right)$, $7.21-7.13\left(\mathrm{~m}, 2 \mathrm{H}, H_{\mathrm{Ar}}\right), 3.60(\mathrm{ddd}, J=15.8,9.7,6.3 \mathrm{~Hz}, 1 \mathrm{H}, \mathrm{CCH})$, $3.23\left(\mathrm{dd}, J=10.4,6.9 \mathrm{~Hz}, 1 \mathrm{H}, \mathrm{CCHCH}_{\mathrm{a}}\right), 3.14(\mathrm{dd}, J=10.4,9.3 \mathrm{~Hz}$, $\left.1 \mathrm{H}, \mathrm{CCHCH}_{\mathrm{a}}{ }^{\prime}\right), 2.98\left(\mathrm{dd}, J=8.4,5.0 \mathrm{~Hz}, 2 \mathrm{H}, \mathrm{SCH}_{2} \mathrm{CH}_{2}\right), 2.52(\mathrm{dt}, J$ $\left.=10.4,5.1 \mathrm{~Hz}, 1 \mathrm{H}, \mathrm{CCHCH}_{\mathrm{b}}\right), 2.36-2.26\left(\mathrm{~m}, 1 \mathrm{H}, \mathrm{CCHCH}_{\mathrm{b}}{ }^{\prime}\right)$; ${ }^{13} \mathrm{C}\left\{{ }^{1} \mathrm{H}\right\}$ NMR $\left(151 \mathrm{MHz}\right.$, methanol- $\left.d_{4}\right) \delta 156.4\left(C_{\mathrm{Ar}}\right), 123.4\left(C_{\mathrm{Ar}} \mathrm{H}\right)$, $115.4\left(\mathrm{br}, C_{\mathrm{Ar}} \mathrm{H}\right), 45.0(\mathrm{CCH}), 37.0\left(C_{\mathrm{b}}\right), 36.0\left(C_{\mathrm{a}}\right), 31.2$ $\left(\mathrm{SCH}_{2} \mathrm{CH}_{2}\right)$; one aromatic carbon is not seen in the ${ }^{13} \mathrm{C}\left\{{ }^{1} \mathrm{H}\right\} \mathrm{NMR}$ spectrum due to peak broadening; HRMS (ESI) calcd for $\mathrm{C}_{11} \mathrm{H}_{13} \mathrm{~N}_{2} \mathrm{~S}^{+}$ $[\mathrm{M}+\mathrm{H}]^{+}$205.0805, found 205.0799; IR $\nu_{\max }$ (film) 2923, 2356, $2348,2158,2034,1420,740 \mathrm{~cm}^{-1}$; mp $242-244{ }^{\circ} \mathrm{C}$.

2-(1,4-Dioxaspiro[4.5]decan-8-yl)-1H-benzimidazole (5e). General procedure $\mathrm{C}$ was followed using 3-methoxy-3-methyl-4,9,12trioxa-1,2-diazadispiro[4.2. $\left.{ }^{8} .2^{5}\right]$ tetradec-1-ene $(51 \mathrm{mg}, 0.2 \mathrm{mmol}, 1.0$ equiv), formaldehyde ( $0.15 \mathrm{~mL}, 37 \mathrm{wt} \%$ in $\mathrm{H}_{2} \mathrm{O}, 2 \mathrm{mmol}, 10$ equiv), and $o$-phenylenediamine ( $32 \mathrm{mg}, 0.3 \mathrm{mmol}, 1.5$ equiv). The crude mixture was purified via flash column chromatography $(10-40 \%$ EtOAc in petroleum ether) to give the titled product as a white solid (41 mg, 80\%): ${ }^{1} \mathrm{H}$ NMR (600 MHz, $\left.\mathrm{CDCl}_{3}\right) \delta 7.57-7.51(\mathrm{~m}, 2 \mathrm{H}$, $\left.H_{\mathrm{Ar}}\right), 7.20\left(\mathrm{dd}, J=6.1,3.1 \mathrm{~Hz}, 2 \mathrm{H}, H_{\mathrm{Ar}}\right), 4.01-3.90(\mathrm{~m}, 4 \mathrm{H}$, $\left.\mathrm{OCH}_{2} \mathrm{CH}_{2} \mathrm{O}\right), 3.02(\mathrm{tt}, J=11.8,3.7 \mathrm{~Hz}, 1 \mathrm{H}, \mathrm{CCH}), 2.21-2.13(\mathrm{~m}$, $\left.2 \mathrm{H}, \mathrm{CHCH}_{\mathrm{a}}+\mathrm{CHCH}_{\mathrm{b}}\right), 2.07-1.97\left(\mathrm{~m}, 2 \mathrm{H}, \mathrm{CHCH}_{\mathrm{a}}{ }^{\prime}+\mathrm{CHCH}_{\mathrm{b}}{ }^{\prime}\right)$, 1.90-1.83 (m, 2H, $\left.\mathrm{CCH}_{\mathrm{c}}+\mathrm{CCH}_{\mathrm{d}}\right), 1.68(\mathrm{td}, J=13.2,4.3 \mathrm{~Hz}, 2 \mathrm{H}$, $\left.\mathrm{CCH}_{\mathrm{c}}{ }^{\prime}+\mathrm{CCH}_{\mathrm{d}}{ }^{\prime}\right) ;{ }^{13} \mathrm{C}\left\{{ }^{1} \mathrm{H}\right\} \operatorname{NMR}\left(151 \mathrm{MHz}, \mathrm{CDCl}_{3}\right) \delta 157.9\left(\mathrm{C}_{\mathrm{Ar}}\right)$, $122.4\left(C_{\mathrm{Ar}} \mathrm{H}\right), 114.8\left(\mathrm{br}, C_{\mathrm{Ar}} \mathrm{H}\right), 108.1(\mathrm{OCO}), 64.5\left(C_{\mathrm{e}}\right), 64.4\left(C_{\mathrm{f}}\right)$, $37.3(\mathrm{CCH}), 34.4\left(C_{\mathrm{c}}+C_{\mathrm{d}}\right), 29.2\left(C_{\mathrm{a}}+C_{\mathrm{b}}\right)$; one aromatic carbon is not seen in the ${ }^{13} \mathrm{C}\left\{{ }^{1} \mathrm{H}\right\}$ NMR spectrum due to peak broadening; HRMS (ESI) calcd for $\mathrm{C}_{15} \mathrm{H}_{19} \mathrm{~N}_{2} \mathrm{O}_{2}^{+}[\mathrm{M}+\mathrm{H}]^{+}$259.1457, found 259.1447; IR $\nu_{\max }$ (film) 2988, 2945, 1678, 1588, 1402, 1344, 1249, 1219, 1089, 1013, 967, 838, $735 \mathrm{~cm}^{-1}$; mp $230-232{ }^{\circ} \mathrm{C}$.

tert-Butyl 4-(1H-Benzimidazol-2-yl)piperidine-1-carboxylate (5f). General procedure $\mathrm{C}$ was followed using tert-butyl 3-methoxy-3methyl-4-oxa-1,2,8-triazaspiro[4.5] dec-1-ene-8-carboxylate $(57 \mathrm{mg}$, $0.2 \mathrm{mmol}, 1.0$ equiv), formaldehyde $\left(0.16 \mathrm{~mL}, 37\right.$ wt \% in $\mathrm{H}_{2} \mathrm{O}$, $2.0 \mathrm{mmol}, 10$ equiv), and $o$-phenylenediamine $(32 \mathrm{mg}, 0.3 \mathrm{mmol}, 1.5$ equiv). The crude mixture was purified via flash column chromatography (10-40\% EtOAc in petroleum ether) to give the titled product as a white solid (30 mg, 49\%): ${ }^{1} \mathrm{H}$ NMR $(600 \mathrm{MHz}$, $\left.\mathrm{CDCl}_{3}\right) \delta 10.02($ br s, $1 \mathrm{H}, \mathrm{NH}), 7.71\left(\right.$ br s, $\left.1 \mathrm{H}, H_{\mathrm{Ar}}\right), 7.41($ br s, $1 \mathrm{H}$, $\left.H_{\mathrm{Ar}}\right), 7.22\left(\mathrm{dd}, J=6.0,3.1 \mathrm{~Hz}, 2 \mathrm{H}, H_{\mathrm{Ar}}\right), 4.23\left(\mathrm{br} \mathrm{s}, 2 \mathrm{H}, \mathrm{NCH}_{\mathrm{c}}+\right.$ $\mathrm{NCH}_{\mathrm{d}}$ ), 3.10 (tt, $\left.J=11.8,3.8 \mathrm{~Hz}, 1 \mathrm{H}, \mathrm{CCH}\right), 2.89$ (br s, $2 \mathrm{H}, \mathrm{NCH}_{\mathrm{c}}{ }^{\prime}$ $\left.+\mathrm{NCH}_{\mathrm{d}}{ }^{\prime}\right), 2.13-2.04\left(\mathrm{~m}, 2 \mathrm{H}, \mathrm{CH}_{\mathrm{a}}+\mathrm{CH}_{\mathrm{b}}\right), 1.85(\mathrm{qd}, J=12.2,4.3$ $\left.\mathrm{Hz}, 2 \mathrm{H}, \mathrm{CH}_{\mathrm{a}}{ }^{\prime}+\mathrm{CH}_{\mathrm{b}}{ }^{\prime}\right), 1.47\left(\mathrm{~s}, 9 \mathrm{H}, \mathrm{C}\left(\mathrm{CH}_{3}\right)_{3}\right) ;{ }^{13} \mathrm{C}\left\{{ }^{1} \mathrm{H}\right\}$ NMR (151 $\left.\mathrm{MHz}, \mathrm{CDCl}_{3}\right) \delta 157.0\left(\mathrm{C}_{\mathrm{Ar}}\right), 154.9\left(\mathrm{NCOOC}\left(\mathrm{CH}_{3}\right)_{3}\right), 143.2(\mathrm{br}$, $\left.C_{\mathrm{Ar}}\right), 122.5\left(\mathrm{br}, C_{\mathrm{Ar}} \mathrm{H}\right), 119.1\left(\mathrm{br}, C_{\mathrm{Ar}} \mathrm{H}\right), 110.7\left(\mathrm{br}, C_{\mathrm{Ar}} \mathrm{H}\right), 80.0$ $\left(\mathrm{C}\left(\mathrm{CH}_{3}\right)_{3}\right), 44.1\left(\mathrm{br}, \mathrm{C}_{\mathrm{c}}+\mathrm{C}_{\mathrm{d}}\right), 37.1(\mathrm{CCH}), 30.9\left(\mathrm{br}, \mathrm{C}_{\mathrm{a}}+\mathrm{C}_{\mathrm{b}}\right), 28.6$ $\left(\mathrm{C}\left(\mathrm{CH}_{3}\right)_{3}\right)$; HRMS (ESI) calcd for $\mathrm{C}_{17} \mathrm{H}_{24} \mathrm{O}_{2} \mathrm{~N}_{3}{ }^{+}[\mathrm{M}+\mathrm{H}]^{+}$ 302.1869, found 302.1869; IR $\nu_{\max }$ (film) 2976, 1692, 1536, 1425, $1366,1272,1231,1166,1123,980,861,768,742 \mathrm{~cm}^{-1}$; mp 226-228 ${ }^{\circ} \mathrm{C}$.

2-[1-(Pyrimidin-2-yl)piperidin-4-yl]-1H-benzimidazole (5g). General procedure $\mathrm{C}$ was followed using 3-methoxy-3-methyl-8(pyrimidin-2-yl)-4-oxa-1,2,8-triazaspiro[4.5] dec-1-ene (55 mg, 0.2 mmol, 1.0 equiv), formaldehyde $\left(0.16 \mathrm{~mL}, 37 \mathrm{wt} \%\right.$ in $\mathrm{H}_{2} \mathrm{O}, 2.0$ mmol, 10 equiv), and o-phenylenediamine $(32 \mathrm{mg}, 0.3 \mathrm{mmol}, 1.5$ equiv). The crude mixture was purified via flash column chromatography $(10-40 \%$ EtOAc in petroleum ether) to give the titled product as a white solid (40 mg, 72\%): ${ }^{1} \mathrm{H}$ NMR $(600 \mathrm{MHz}$, $\left.\mathrm{CDCl}_{3}\right) \delta 9.75$ (br s, $\left.1 \mathrm{H}, \mathrm{NH}\right), 8.30\left(\mathrm{~d}, J=4.6 \mathrm{~Hz}, 2 \mathrm{H}, H_{\text {pyrimidine }}\right)$, $7.54\left(\right.$ br s, $\left.2 \mathrm{H}, H_{\mathrm{Ar}}\right), 7.22\left(\mathrm{dd}, J=5.9,3.1 \mathrm{~Hz}, 2 \mathrm{H}, H_{\mathrm{Ar}}\right), 6.47(\mathrm{t}, J=$ $\left.4.6 \mathrm{~Hz}, 1 \mathrm{H}, H_{\text {pyrimidine }}\right), 4.86\left(\mathrm{~d}, J=13.5 \mathrm{~Hz}, 2 \mathrm{H}, \mathrm{NCH}_{\mathrm{c}}+\mathrm{NCH}_{\mathrm{d}}\right)$, $3.22(\mathrm{tt}, J=11.8,3.7 \mathrm{~Hz}, 1 \mathrm{H}, \mathrm{CCH}), 3.10-2.99\left(\mathrm{~m}, 2 \mathrm{H}, \mathrm{NCH}_{\mathrm{c}}{ }^{\prime}+\right.$ $\left.\mathrm{NCH}_{\mathrm{d}}{ }^{\prime}\right), 2.19$ (d, $J=11.3 \mathrm{~Hz}, 2 \mathrm{H}, \mathrm{CH}_{\mathrm{a}}+\mathrm{CH}_{\mathrm{b}}$ ), 1.92 (qd, $J=12.4$, $\left.3.9 \mathrm{~Hz}, 2 \mathrm{H}, \mathrm{CH}_{\mathrm{a}}{ }^{\prime}+\mathrm{CH}_{\mathrm{b}}{ }^{\prime}\right) ;{ }^{13} \mathrm{C}\left\{{ }^{1} \mathrm{H}\right\}$ NMR $\left(151 \mathrm{MHz}, \mathrm{CDCl}_{3}\right) \delta$ $161.7\left(C_{\text {pyrimidine }}\right), 157.9\left(C_{\text {pyrimidine }} \mathrm{H}\right), 157.2\left(C_{\mathrm{Ar}}\right), 122.6\left(\mathrm{br}, C_{\mathrm{Ar}} \mathrm{H}\right)$, $110.0\left(C_{\text {pyrimidine }} \mathrm{H}\right), 43.9\left(C_{\mathrm{c}}+C_{\mathrm{d}}\right), 37.3(\mathrm{CCH}), 30.7\left(C_{\mathrm{a}}+C_{\mathrm{b}}\right)$; two aromatic carbons are not seen in the ${ }^{13} \mathrm{C}\left\{{ }^{1} \mathrm{H}\right\}$ NMR spectrum due to peak broadening; HRMS (ESI) calcd for $\mathrm{C}_{16} \mathrm{H}_{18} \mathrm{~N}_{5}^{+}[\mathrm{M}+\mathrm{H}]^{+}$ 280.1557, found 280.1546; IR $\nu_{\max }$ (film) 2936, 2346, 1982, 1584, $1541,1518,1481,1456,1426,1358,1304,1272,1233,1105,1050$, 977, 798, $741 \mathrm{~cm}^{-1}$; mp $222-224{ }^{\circ} \mathrm{C}$.

2-(Adamantan-2-yl)-1H-benzimidazole (5h). General procedure $\mathrm{C}$ was followed using $5^{\prime}$-methoxy-5'-methyl-5' $\mathrm{H}$-spiro[adamantane$2,2^{\prime}$-[1,3,4] oxadiazole] (47 mg, $0.2 \mathrm{mmol}, 1.0$ equiv), formaldehyde $\left(0.15 \mathrm{~mL}, 37\right.$ wt $\%$ in $\mathrm{H}_{2} \mathrm{O}, 2 \mathrm{mmol}, 10$ equiv), and $o$ phenylenediamine ( $32 \mathrm{mg}, 0.3 \mathrm{mmol}, 1.5$ equiv). The crude mixture was purified via flash column chromatography $(10-40 \%$ EtOAc in petroleum ether) to give the titled product as a dark yellow solid (40 mg, 79\%): ${ }^{1} \mathrm{H}$ NMR (600 MHz, $\left.\mathrm{CDCl}_{3}\right) \delta 7.57$ (br s, $\left.2 \mathrm{H}, \mathrm{H}_{\mathrm{Ar}}\right), 7.21$ $\left(\mathrm{dd}, J=6.0,3.1 \mathrm{~Hz}, 2 \mathrm{H}, H_{\mathrm{Ar}}\right), 3.27(\mathrm{~s}, 1 \mathrm{H}, \mathrm{CCH}), 2.66-2.59(\mathrm{~m}, 2 \mathrm{H}$, $\left.\mathrm{CH}_{\mathrm{a}}\right), 2.08-1.94\left(\mathrm{~m}, 7 \mathrm{H}, \mathrm{CH}_{\mathrm{c}}+\mathrm{C}_{\mathrm{b}}\right), 1.87-1.86\left(\mathrm{~m}, 1 \mathrm{H}, \mathrm{CH}_{\mathrm{c}}{ }^{\prime}\right), 1.83$ (br s, $\left.2 \mathrm{H}, \mathrm{C}_{\mathrm{d}}\right), 1.77-1.70\left(\mathrm{~m}, 2 \mathrm{H}, \mathrm{C}_{\mathrm{b}}\right) ;{ }^{13} \mathrm{C}\left\{{ }^{1} \mathrm{H}\right\}$ NMR $(151 \mathrm{MHz}$, $\left.\mathrm{CDCl}_{3}\right) \delta 157.2\left(\mathrm{C}_{\mathrm{Ar}}\right), 122.3\left(\mathrm{C}_{\mathrm{Ar}} \mathrm{H}\right), 44.6(\mathrm{CCH}), 38.4\left(C_{\mathrm{b}}\right), 37.6$ $\left(C_{\mathrm{d}}\right), 33.1\left(C_{\mathrm{b}}{ }^{\prime}\right), 31.0\left(C_{\mathrm{a}}\right), 27.8\left(C_{\mathrm{c}}\right), 27.7\left(C_{\mathrm{c}}{ }^{\prime}\right)$; two aromatic carbons are not seen in the ${ }^{13} \mathrm{C}\left\{{ }^{1} \mathrm{H}\right\}$ NMR spectrum due to peak broadening; HRMS (ESI) calcd for $\mathrm{C}_{17} \mathrm{H}_{21} \mathrm{~N}_{2}{ }^{+}[\mathrm{M}+\mathrm{H}]^{+}$253.1705, found 253.1700; IR $\nu_{\max }$ (film) 2922, 1422, 1275, 1009, 993, 743 $\mathrm{cm}^{-1}$; mp 244-246 ${ }^{\circ} \mathrm{C}$.

2-Cyclobutyl-1H-benzimidazole (5i). General procedure $\mathrm{C}$ was followed using 7-methoxy-7-methyl-8-oxa-5,6-diazaspiro[3.4] oct-5ene $(31 \mathrm{mg}, 0.2 \mathrm{mmol}, 1.0$ equiv), formaldehyde $(0.16 \mathrm{~mL}, 37 \mathrm{wt}$ $\%$ in $\mathrm{H}_{2} \mathrm{O}, 2.0 \mathrm{mmol}, 10$ equiv), and $o$-phenylenediamine ( $32 \mathrm{mg}, 0.3$ mmol, 1.5 equiv). The crude mixture was purified via flash column chromatography $(10-40 \%$ EtOAc in petroleum ether) to give the titled product as a white solid $(20 \mathrm{mg}, 59 \%):{ }^{1} \mathrm{H}$ NMR $(600 \mathrm{MHz}$, $\left.\mathrm{CDCl}_{3}\right) \delta 7.56\left(\mathrm{dd}, J=6.0,3.2 \mathrm{~Hz}, 2 \mathrm{H}, H_{\mathrm{Ar}}\right), 7.21(\mathrm{dd}, J=6.0,3.1$ $\left.\mathrm{Hz}, 2 \mathrm{H}, \mathrm{H}_{\mathrm{Ar}}\right), 3.80(\mathrm{p}, J=8.7 \mathrm{~Hz}, 1 \mathrm{H}, \mathrm{CCH}), 2.58-2.47\left(\mathrm{~m}, 2 \mathrm{H}, \mathrm{CH}_{\mathrm{a}}\right.$ $\left.+\mathrm{CH}_{\mathrm{b}}\right), 2.47-2.38\left(\mathrm{~m}, 2 \mathrm{H}, \mathrm{CH}_{\mathrm{a}}{ }^{\prime}+\mathrm{CH}_{\mathrm{b}}{ }^{\prime}\right), 2.14-2.01\left(\mathrm{~m}, 1 \mathrm{H}, \mathrm{CH}_{\mathrm{c}}\right)$, 1.99-1.91 (m, 1H, $\left.\mathrm{CH}_{\mathrm{c}}{ }^{\prime}\right) ;{ }^{13} \mathrm{C}\left\{{ }^{1} \mathrm{H}\right\}$ NMR $\left(151 \mathrm{MHz}, \mathrm{CDCl}_{3}\right) \delta$ $158.1\left(C_{\mathrm{Ar}}\right), 138.6\left(\mathrm{br}, C_{\mathrm{Ar}}\right), 122.3\left(C_{\mathrm{Ar}} \mathrm{H}\right), 114.8\left(\mathrm{br}, C_{\mathrm{Ar}} \mathrm{H}\right), 34.3$ $(\mathrm{CCH}), 28.2\left(\mathrm{C}_{\mathrm{a}}+\mathrm{C}_{\mathrm{b}}\right), 18.8\left(\mathrm{C}_{\mathrm{c}}\right)$; HRMS (ESI) calcd for $\mathrm{C}_{11} \mathrm{H}_{13} \mathrm{~N}_{2}{ }^{+}$ $[\mathrm{M}+\mathrm{H}]^{+} 173.1073$, found 173.1069; IR $\nu_{\max }$ (film) 2942, 1537, $1455,1419,1328,1272,982,740 \mathrm{~cm}^{-1}$; $\mathrm{mp} 186-188^{\circ} \mathrm{C}$. The data presented are consistent with literature precedent. ${ }^{46}$

2-[1-(Pyridin-4-yl)propan-2-yl]-1H-benzimidazole (5I). General procedure $\mathrm{C}$ was followed using 2-methoxy-2,5-dimethyl-5-(pyridin4-ylmethyl)-2,5-dihydro-1,3,4-oxadiazole (44 mg, $0.2 \mathrm{mmol}, 1.0$ equiv), formaldehyde $\left(0.16 \mathrm{~mL}, 37 \mathrm{wt} \%\right.$ in $\mathrm{H}_{2} \mathrm{O}, 2.0 \mathrm{mmol}, 10$ equiv), and $o$-phenylenediamine ( $32 \mathrm{mg}, 0.3 \mathrm{mmol}, 1.5$ equiv). The crude mixture was purified via flash column chromatography (10$100 \%$ EtOAc in petroleum ether) to give the titled product as a yellow solid $(23 \mathrm{mg}, 48 \%):{ }^{1} \mathrm{H}$ NMR $\left(600 \mathrm{MHz}, \mathrm{CDCl}_{3}\right) \delta 10.14$ (br s, $1 \mathrm{H}$, $\mathrm{NH}), 8.40-8.36\left(\mathrm{~m}, 2 \mathrm{H}, H_{\text {Pyridine }}\right), 7.73$ (br s, $\left.1 \mathrm{H}, H_{\mathrm{Ar}}\right), 7.34$ (br s, $\left.1 \mathrm{H}, H_{\mathrm{Ar}}\right), 7.23\left(\mathrm{~s}, 2 \mathrm{H}, H_{\mathrm{Ar}}\right), 7.00-6.98\left(\mathrm{~m}, 2 \mathrm{H}, H_{\text {Pyridine }}\right), 3.35(\mathrm{p}, J=$ $7.0 \mathrm{~Hz}, 1 \mathrm{H}, \mathrm{CCH}), 3.28\left(\mathrm{dd}, J=13.4,7.5 \mathrm{~Hz}, 1 \mathrm{H}, \mathrm{CHCH}_{\mathrm{a}}\right), 3.00(\mathrm{dd}$, $\left.J=13.4,6.8 \mathrm{~Hz}, 1 \mathrm{H}, \mathrm{CHCH}_{\mathrm{a}}{ }^{\prime}\right), 1.47\left(\mathrm{~d}, J=6.9 \mathrm{~Hz}, 3 \mathrm{H}, \mathrm{CH}_{3}\right)$; ${ }^{13} \mathrm{C}\left\{{ }^{1} \mathrm{H}\right\}$ NMR $\left(151 \mathrm{MHz}, \mathrm{CDCl}_{3}\right) \delta 157.3\left(C_{\mathrm{Ar}}\right), 149.5\left(C_{\text {Pyridine }} \mathrm{H}\right)$, $148.8\left(C_{\text {Pyridine }}\right), 143.1\left(\mathrm{br}, C_{\mathrm{Ar}}\right), 124.5\left(C_{\text {Pyridine }} \mathrm{H}\right), 122.3\left(\mathrm{br}, C_{\mathrm{Ar}} \mathrm{H}\right)$, $110.5\left(\mathrm{br}, \mathrm{C}_{\mathrm{Ar}} \mathrm{H}\right), 41.8\left(\mathrm{C}_{\mathrm{a}}\right), 35.9(\mathrm{CCH}), 19.4\left(\mathrm{CH}_{3}\right)$. HRMS (ESI) calcd for $\mathrm{C}_{15} \mathrm{H}_{16} \mathrm{~N}_{3}{ }^{+}[\mathrm{M}+\mathrm{H}]^{+}$238.1344, found 238.1344; IR $\nu_{\max }$ (film) $3051,2969,1603,1559,1535,1484,1454,1419,1328,1272$, $1219,1110,1070,1043,993,907,843,795,768,747 \mathrm{~cm}^{-1}$; mp 188$190{ }^{\circ} \mathrm{C}$.

2-[1-(Furan-2-yl)propan-2-yl]-1H-benzimidazole (5m). General procedure $\mathrm{C}$ was followed using 2-(furan-2-ylmethyl)-5-methoxy2,5-dimethyl-2,5-dihydro-1,3,4-oxadiazole $(42 \mathrm{mg}, 0.2 \mathrm{mmol}, 1.0$ equiv), formaldehyde ( $0.15 \mathrm{~mL}, 37 \mathrm{wt} \%$ in $\mathrm{H}_{2} \mathrm{O}, 2 \mathrm{mmol}, 10$ equiv), and $o$-phenylenediamine $(32 \mathrm{mg}, 0.3 \mathrm{mmol}, 1.5$ equiv). The crude mixture was purified via flash column chromatography $(10-40 \%$ EtOAc in petroleum ether) to give the titled product as a white solid (34 mg, 75\%): ${ }^{1} \mathrm{H}$ NMR (600 MHz, $\left.\mathrm{CDCl}_{3}\right) \delta 7.53$ (dd, $J=6.1,3.2$ $\left.\mathrm{Hz}, 2 \mathrm{H}, H_{\mathrm{Ar}}\right), 7.30-7.27\left(\mathrm{~m}, 1 \mathrm{H}, H_{\text {Furan }}\right), 7.23-7.18\left(\mathrm{~m}, 2 \mathrm{H}, H_{\mathrm{Ar}}\right)$, $6.24\left(\mathrm{dd}, J=3.2,1.9 \mathrm{~Hz}, 1 \mathrm{H}, H_{\text {Furan }}\right), 5.96\left(\mathrm{~d}, J=3.2 \mathrm{~Hz}, 1 \mathrm{H}, H_{\text {Furan }}\right)$, $3.51(\mathrm{~h}, J=7.1 \mathrm{~Hz}, 1 \mathrm{H}, \mathrm{CCH}), 3.24(\mathrm{dd}, J=15.0,7.2 \mathrm{~Hz}, 1 \mathrm{H}$, $\left.\mathrm{CHCH}_{\mathrm{a}}\right), 3.06\left(\mathrm{dd}, J=15.0,7.2 \mathrm{~Hz}, 1 \mathrm{H}, \mathrm{CHCH}_{\mathrm{a}}{ }^{\prime}\right), 1.48(\mathrm{~d}, J=7.0$ $\left.\mathrm{Hz}, 3 \mathrm{H}, \mathrm{CHCH}_{3}\right) ;{ }^{13} \mathrm{C}\left\{{ }^{1} \mathrm{H}\right\}$ NMR $\left(151 \mathrm{MHz} \mathrm{CDCl}_{3}\right) \delta 158.1$ 
$\left(C_{\text {Furan }}\right), 153.3\left(C_{\mathrm{Ar}}\right), 141.5\left(C_{\text {Furan }} \mathrm{H}\right), 122.4\left(C_{\mathrm{Ar}} \mathrm{H}\right), 115.1$ (br, $\left.C_{\mathrm{Ar}} \mathrm{H}\right), 110.5\left(C_{\text {Furan }} \mathrm{H}\right)$, 107.1 $\left(C_{\text {Furan }} \mathrm{H}\right), 34.6\left(C_{\mathrm{a}}\right), 34.1(\mathrm{CCH}), 19.3$ $\left(\mathrm{CH}_{3}\right)$; one aromatic carbon is not seen in the ${ }^{13} \mathrm{C}\left\{{ }^{1} \mathrm{H}\right\}$ NMR spectrum; HRMS (ESI+): $m / z$ calcd for $\mathrm{C}_{14} \mathrm{H}_{15} \mathrm{~N}_{2} \mathrm{O}^{+}[\mathrm{M}+\mathrm{H}]^{+}$ 227.1177, found 227.1184; IR $\nu_{\max }$ (film) 2921, 1423, 1275, 1009, 993, 932, $743 \mathrm{~cm}^{-1}$; mp $180-182{ }^{\circ} \mathrm{C}$.

2-(Hex-5-en-2-yl)-1H-benzimidazole (5n). General procedure $\mathrm{C}$ was followed using 2-(but-3-en-1-yl)-5-methoxy-2,5-dimethyl-2,5dihydro-1,3,4-oxadiazole ( $37 \mathrm{mg}, 0.2 \mathrm{mmol}, 1.0$ equiv), formaldehyde (0.16 mL, 37 wt \% in $\mathrm{H}_{2} \mathrm{O}, 2.0 \mathrm{mmol}, 10$ equiv) and $o$ phenylenediamine ( $32 \mathrm{mg}, 0.3 \mathrm{mmol}, 1.5$ equiv). The crude mixture was purified via flash column chromatography $(10-40 \%$ EtOAc in petroleum ether) to give the titled product as a white solid $(29 \mathrm{mg}$, $73 \%) .{ }^{1} \mathrm{H}$ NMR $\left(600 \mathrm{MHz}^{\circ} \mathrm{CDCl}_{3}\right) \delta 10.18(\mathrm{br} \mathrm{s}, 1 \mathrm{H}, \mathrm{NH}), 7.55$ (br s, $\left.2 \mathrm{H}, H_{\mathrm{Ar}}\right), 7.21\left(\mathrm{dd}, J=6.0,3.1 \mathrm{~Hz}, 2 \mathrm{H}, H_{\mathrm{Ar}}\right), 5.75(\mathrm{ddt}, J=17.0$, $\left.10.2,6.6 \mathrm{~Hz}, 1 \mathrm{H}, \mathrm{CH}_{2}=\mathrm{CH}\right), 4.97\left(\mathrm{dd}, J=17.0,1.8 \mathrm{~Hz}, 1 \mathrm{H}, H_{\mathrm{a}}\right)$, $4.93\left(\mathrm{dd}, J=10.2,1.8 \mathrm{~Hz}, 1 \mathrm{H}, H_{\mathrm{b}}\right), 3.14(\mathrm{~h}, J=7.0 \mathrm{~Hz}, 1 \mathrm{H}, \mathrm{CCH})$, 2.14-2.05 (m, 2H, $\left.\mathrm{CH}_{2}=\mathrm{CHCH}_{2}\right), 2.04-1.97\left(\mathrm{~m}, 1 \mathrm{H}, \mathrm{CCHCH}_{\mathrm{c}}\right)$, $1.85-1.77\left(\mathrm{~m}, 1 \mathrm{H}, \mathrm{CCHCH}_{\mathrm{c}}{ }^{\prime}\right), 1.45\left(\mathrm{~d}, J=7.1 \mathrm{~Hz}, 3 \mathrm{H}, \mathrm{CH}_{3}\right)$; ${ }^{13} \mathrm{C}\left\{{ }^{1} \mathrm{H}\right\} \operatorname{NMR}\left(151 \mathrm{MHz}, \mathrm{CDCl}_{3}\right) \delta 159.3\left(\mathrm{C}_{\mathrm{Ar}}\right), 137.9\left(\mathrm{CH}_{2}=\mathrm{CH}\right)$, $122.3\left(\mathrm{C}_{\mathrm{Ar}} \mathrm{H}\right), 115.3\left(\mathrm{CH}_{2}=\mathrm{CH}\right), 35.6\left(C_{\mathrm{c}}\right), 34.2(\mathrm{CCH}), 31.6$ $\left(\mathrm{CH}_{2}=\mathrm{CHCH}_{2}\right), 19.8\left(\mathrm{CH}_{3}\right)$; two aromatic carbons are not seen in the ${ }^{13} \mathrm{C}\left\{{ }^{1} \mathrm{H}\right\}$ NMR spectrum; HRMS (ESI) calcd for $\mathrm{C}_{13} \mathrm{H}_{17} \mathrm{~N}_{2}{ }^{+}[\mathrm{M}+$ $\mathrm{H}]^{+}$201.1386, found 201.1379; IR $\nu_{\max }$ (film) 3074, 2968, 2932, $2736,1818,1640,1538,1454,1426,1330,1272,989,906,745,729$ $\mathrm{cm}^{-1}$; mp $182-185^{\circ} \mathrm{C}$.

2-(4,4-Dimethylhept-6-yn-2-yl)-1H-benzimidazole (5o). General procedure $\mathrm{C}$ was followed using 2-(2,2-dimethylpent-4-yn-1-yl)-5methoxy-2,5-dimethyl-2,5-dihydro-1,3,4-oxadiazole (45 mg, 0.2 mmol, 1.0 equiv), formaldehyde $\left(0.16 \mathrm{~mL}, 37\right.$ wt $\%$ in $\mathrm{H}_{2} \mathrm{O}, 2.0$ mmol, 10 equiv), and o-phenylenediamine $(32 \mathrm{mg}, 0.3 \mathrm{mmol}, 1.5$ equiv). The crude mixture was purified via flash column chromatography $(10-40 \%$ EtOAc in petroleum ether) to give the titled product as a white solid (19 mg, 39\%): ${ }^{1} \mathrm{H}$ NMR $(600 \mathrm{MHz}$, $\left.\mathrm{CDCl}_{3}\right) \delta 9.68($ br s, $1 \mathrm{H}, \mathrm{NH}), 7.71\left(\right.$ br s, $\left.1 \mathrm{H}, \mathrm{H}_{\mathrm{Ar}}\right), 7.38($ br s, $1 \mathrm{H}$, $\left.H_{\mathrm{Ar}}\right), 7.21\left(\mathrm{dd}, J=6.2,2.9 \mathrm{~Hz}, 2 \mathrm{H}, H_{\mathrm{Ar}}\right), 3.29-3.21(\mathrm{~m}, 1 \mathrm{H}$, $\left.\mathrm{CCHCH}_{3}\right), 2.12\left(\mathrm{dd}, J=14.4,8.9 \mathrm{~Hz}, 1 \mathrm{H}, \mathrm{CHCCH}_{\mathrm{b}}\right), 2.07(\mathrm{dd}, J=$ 16.2, $\left.3.2 \mathrm{~Hz}, 1 \mathrm{H}, \mathrm{CHCCH}_{\mathrm{a}}\right), 2.02-1.93\left(\mathrm{~m}, 2 \mathrm{H}, \mathrm{CHCCH}_{2}+\right.$ $\left.\mathrm{CHCCH}_{\mathrm{a}}{ }^{\prime}\right), 1.72\left(\mathrm{dd}, J=14.4,4.0 \mathrm{~Hz}, 1 \mathrm{H}, \mathrm{CHCCH}_{\mathrm{b}}{ }^{\prime}\right), 1.44(\mathrm{~d}, J=$ $\left.7.1 \mathrm{~Hz}, 3 \mathrm{H}, \mathrm{CHCH}_{3}\right), 0.92\left(\mathrm{~d}, J=17.9 \mathrm{~Hz}, 6 \mathrm{H}, \mathrm{C}\left(\mathrm{CH}_{3}\right)_{2}\right) ;{ }^{13} \mathrm{C}\left\{{ }^{1} \mathrm{H}\right\}$ NMR $\left(151 \mathrm{MHz}, \mathrm{CDCl}_{3}\right) \delta 159.7\left(C_{\mathrm{Ar}}\right), 122.3\left(\mathrm{br}, \mathrm{C}_{\mathrm{Ar}} \mathrm{H}\right), 110.7(\mathrm{br}$, $\left.\mathrm{C}_{\mathrm{Ar}} \mathrm{H}\right), 82.6\left(\mathrm{CHCCH}_{2}\right), \quad 70.5\left(\mathrm{CHCCH}_{2}\right), 47.1\left(\mathrm{C}_{\mathrm{b}}\right), 34.0$ $\left(\mathrm{C}\left(\mathrm{CH}_{3}\right)_{2}\right), 31.9\left(\mathrm{C}_{\mathrm{a}}\right), 31.2\left(\mathrm{CCHCH}_{3}\right), 27.3\left(\mathrm{C}\left(\mathrm{CH}_{3}\right)_{2}\right), 27.1$ $\left(\mathrm{C}\left(\mathrm{CH}_{3}\right)_{2}\right), 23.3\left(\mathrm{CCHCH}_{3}\right)$; one aromatic carbon is not seen in the ${ }^{13} \mathrm{C}\left\{{ }^{1} \mathrm{H}\right\}$ NMR spectrum; HRMS (ESI) calcd for $\mathrm{C}_{16} \mathrm{H}_{21} \mathrm{~N}_{2}{ }^{+}[\mathrm{M}+$ $\mathrm{H}]^{+}$241.1699, found 241.1690; IR $\nu_{\max }$ (film) 3311, 2965, 2752, $2367,1538,1453,1424,1335,1269,994,746 \mathrm{~cm}^{-1}$; mp 191-192 ${ }^{\circ} \mathrm{C}$.

2-(1-Cyclopropylethyl)-1H-benzimidazole (5p). General procedure $\mathrm{C}$ was followed using 2-cyclopropyl-5-methoxy-2,5-dimethyl-2,5dihydro-1,3,4-oxadiazole ( $34 \mathrm{mg}, 0.2 \mathrm{mmol}, 1.0$ equiv), formaldehyde (0.16 mL, 37 wt \% in $\mathrm{H}_{2} \mathrm{O}, 2.0 \mathrm{mmol}, 10$ equiv), and $o$ phenylenediamine ( $32 \mathrm{mg}, 0.3 \mathrm{mmol}, 1.5$ equiv). The crude mixture was purified via flash column chromatography $(10-40 \%$ EtOAc in petroleum ether) to give the titled product as a white solid $(26 \mathrm{mg}$, 69\%): ${ }^{1} \mathrm{H}$ NMR $\left(600 \mathrm{MHz}, \mathrm{CDCl}_{3}\right) \delta 9.49(\mathrm{br} \mathrm{s}, 1 \mathrm{H}, \mathrm{NH}), 7.73(\mathrm{br}$ s, $\left.1 \mathrm{H}, H_{\mathrm{Ar}}\right), 7.41\left(\mathrm{br} \mathrm{s}, 1 \mathrm{H}, H_{\mathrm{Ar}}\right), 7.22\left(\mathrm{dd}, J=6.1,3.1 \mathrm{~Hz}, 2 \mathrm{H}, H_{\mathrm{Ar}}\right)$, $2.36(\mathrm{dq}, J=9.6,7.0 \mathrm{~Hz}, 1 \mathrm{H}, \mathrm{CCH}), 1.56\left(\mathrm{~d}, J=7.0 \mathrm{~Hz}, 3 \mathrm{H}, \mathrm{CH}_{3}\right)$, 1.10 (dddd, $J=13.0,9.6,8.0,4.9 \mathrm{~Hz}, 1 \mathrm{H}, \mathrm{CHCHCH}_{2}$ ), 0.71-0.63 $\left(\mathrm{m}, 2 \mathrm{H}, \mathrm{CH}_{\mathrm{a}}+\mathrm{CH}_{\mathrm{a}}{ }^{\prime}\right), 0.44-0.38\left(\mathrm{~m}, 1 \mathrm{H}, \mathrm{CH}_{\mathrm{b}}\right), 0.36-0.30(\mathrm{~m}, 1 \mathrm{H}$, $\left.\mathrm{CH}_{\mathrm{b}}{ }^{\prime}\right) ;{ }^{13} \mathrm{C}\left\{{ }^{\mathrm{l}} \mathrm{H}\right\}$ NMR $\left(151 \mathrm{MHz}, \mathrm{CDCl}_{3}\right) \delta 158.7\left(\mathrm{C}_{\mathrm{Ar}}\right), 143.4(\mathrm{br}$, $\left.C_{\mathrm{Ar}}\right), 133.5\left(\mathrm{br}, C_{\mathrm{Ar}}\right), 122.3\left(C_{\mathrm{Ar}} \mathrm{H}\right), 119.4\left(\mathrm{br}, C_{\mathrm{Ar}} \mathrm{H}\right), 110.4(\mathrm{br}$, $\left.C_{\mathrm{Ar}} \mathrm{H}\right), 39.5(\mathrm{CCH}), 19.0\left(\mathrm{CH}_{3}\right), 16.6(\mathrm{CCHCH}), 4.8\left(\mathrm{C}_{\mathrm{a}}\right), 4.4\left(\mathrm{C}_{\mathrm{b}}\right)$; HRMS (ESI) calcd for $\mathrm{C}_{12} \mathrm{H}_{15} \mathrm{~N}_{2}{ }^{+}[\mathrm{M}+\mathrm{H}]^{+}$187.1230, found 187.1221; IR $\nu_{\max }$ (film) 2969, 2317, 2135, 1456, 1414, 1274, 1076, $744 \mathrm{~cm}^{-1}$; mp $187-189^{\circ} \mathrm{C}$.

Synthesis of Aldehydes from Aryl Hydrazones. General Procedure D for the Synthesis of Aldehydes from Aryl Hydrazones. Conditioning phase: A solution of triethylamine in $\mathrm{MeOH}(5 \mathrm{~mL}$, $20 \% \mathrm{v} / \mathrm{v}$ ) was passed through the column reactor (Omnifit column, $6.6 \mathrm{~mm}$ i.d. $\times 50 \mathrm{~mm}$ length $)$, packed with activated $\mathrm{MnO}_{2}(1.0 \mathrm{~g})$, at a flow rate of $1.0 \mathrm{~mL} / \mathrm{min}$ for $5 \mathrm{~min}$ (phase 1 ), and the reactor output was monitored using a Flow-IR device. The flow was switched to EtOAc for 10 min (phase 2). The column was then ready for the generation of the diazo compound.

Generation phase: A solution of hydrazone $(2 \mathrm{mmol}, 0.1 \mathrm{M})$ in EtOAc $(20 \mathrm{~mL})$ was passed through a conditioned column reactor (Omnifit column, $6.6 \mathrm{~mm}$ i.d. $\times 50 \mathrm{~mm}$ length) (phase 3) at a flow rate of $1.0 \mathrm{~mL} / \mathrm{min}$. When the Mettler Toledo FlowIR instrument (SiComp head) showed that the intensity of the diazo peak (region $2060-2080 \mathrm{~cm}^{-1}$ ) was stable, $4 \mathrm{~mL}$ of the stream of diazo was combined with $4 \mathrm{~mL}$ of aqueous formaldehyde (37 wt \%, $1.0 \mathrm{~mL} /$ min) in a UQ5102 Uniqsis Glass Static Mixer at room temperature. The output stream was extracted with extra EtOAc $(20 \mathrm{~mL} \times 2)$ and washed with water $(20 \mathrm{~mL})$. The combined organic phase was dried over $\mathrm{MgSO}_{4}$, concentrated under vacuum, and purified over silica gel using appropriate eluent combinations to yield the desired aldehyde.

2-(4-Chlorophenyl)acetaldehyde (7a). General procedure D was followed using (4-chlorobenzylidene)hydrazine (0.1 M in EtOAc, 1.0 $\mathrm{mL} / \mathrm{min}$ ) and formaldehyde ( $37 \mathrm{wt} \%$ in $\mathrm{H}_{2} \mathrm{O}, 1.0 \mathrm{~mL} / \mathrm{min}$ ). The crude mixture was purified via flash column chromatography $(0-20 \%$ EtOAc in petroleum ether) to give the titled product as a colorless liquid (33 mg, 53\%): ${ }^{1} \mathrm{H}$ NMR (600 MHz, $\left.\mathrm{CDCl}_{3}\right) \delta 9.74(\mathrm{t}, J=2.1$ $\mathrm{Hz}, 1 \mathrm{H}, H \mathrm{CO}), 7.34\left(\mathrm{~d}, J=8.4 \mathrm{~Hz}, 2 \mathrm{H}, H_{\mathrm{Ar}}\right), 7.15(\mathrm{~d}, J=8.3 \mathrm{~Hz}, 2 \mathrm{H}$, $\left.H_{\text {Ar }}\right), 3.68\left(\mathrm{~d}, J=2.1 \mathrm{~Hz}, 2 \mathrm{H}, \mathrm{CH}_{2}\right) ;{ }^{13} \mathrm{C}\left\{{ }^{1} \mathrm{H}\right\}$ NMR $(151 \mathrm{MHz}$, $\left.\mathrm{CDCl}_{3}\right) \delta 198.8(\mathrm{HCO}), 133.6\left(C_{\mathrm{Ar}}\right), 131.1\left(C_{\mathrm{Ar}} \mathrm{H}\right), 130.4\left(C_{\mathrm{Ar}}\right)$, $129.3\left(C_{\mathrm{Ar}} \mathrm{H}\right), 49.9\left(\mathrm{CH}_{2}\right)$; LRMS (ESI, $\left.m / z\right) 155.3\left(\left[{ }^{35} \mathrm{M}+\mathrm{H}\right]^{+}\right.$, 100). The data presented are consistent with literature precedent. ${ }^{47}$

2-(3-Bromophenyl)acetaldehyde (7b). General procedure D was followed using (3-bromobenzylidene)hydrazine (0.1 M in EtOAc, 1.0 $\mathrm{mL} / \mathrm{min}$ ) and formaldehyde ( $37 \mathrm{wt} \%$ in $\mathrm{H}_{2} \mathrm{O}, 1.0 \mathrm{~mL} / \mathrm{min}$ ). The crude mixture was purified via flash column chromatography $(0-20 \%$ EtOAc in petroleum ether) to give the titled product as a colorless liquid (46 mg, 58\%): ${ }^{1} \mathrm{H} \mathrm{NMR}\left(600 \mathrm{MHz}, \mathrm{CDCl}_{3}\right) \delta 9.75(\mathrm{t}, J=2.1$ $\mathrm{Hz}, 1 \mathrm{H}, H \mathrm{CO}), 7.45\left(\mathrm{~d}, J=8.0 \mathrm{~Hz}, 1 \mathrm{H}, H_{\mathrm{Ar}}\right), 7.39(\mathrm{t}, J=1.8 \mathrm{~Hz}, 1 \mathrm{H}$, $\left.H_{\mathrm{Ar}}\right), 7.24\left(\mathrm{t}, J=7.9 \mathrm{~Hz}, 1 \mathrm{H}, H_{\mathrm{Ar}}\right), 7.15\left(\mathrm{~d}, J=7.6 \mathrm{~Hz}, 1 \mathrm{H}, H_{\mathrm{Ar}}\right), 3.68$ $\left(\mathrm{d}, J=2.2 \mathrm{~Hz}, 2 \mathrm{H}, \mathrm{CH}_{2}\right) ;{ }^{13} \mathrm{C}\left\{{ }^{1} \mathrm{H}\right\} \mathrm{NMR}\left(101 \mathrm{MHz}, \mathrm{CDCl}_{3}\right) \delta 198.5$ (HCO), $134.2\left(C_{\mathrm{Ar}}\right), 132.8\left(C_{\mathrm{Ar}} \mathrm{H}\right), 130.8\left(C_{\mathrm{Ar}} \mathrm{H}\right), 130.6\left(C_{\mathrm{Ar}} \mathrm{H}\right)$, $128.4\left(C_{\mathrm{Ar}} \mathrm{H}\right), 123.1\left(C_{\mathrm{Ar}}\right), 50.1\left(\mathrm{CH}_{2}\right)$; LRMS (ESI, $\left.m / z\right) 199.1$ $\left(\left[{ }^{79} \mathrm{M}+\mathrm{H}\right]^{+}, 100\right)$; IR $\nu_{\max }$ (film) $2827,1723,1568,1474,1427$, $1072,782,692 \mathrm{~cm}^{-1}$. The data presented are consistent with literature precedent. ${ }^{46}$

2-(o-Tolyl)acetaldehyde (7c). General procedure D was followed using (2-methylbenzylidene)hydrazine $(0.1 \mathrm{M}$ in EtOAc, $1.0 \mathrm{~mL} /$ min) and formaldehyde ( 37 wt $\%$ in $\left.\mathrm{H}_{2} \mathrm{O}, 1.0 \mathrm{~mL} / \mathrm{min}\right)$. The crude mixture was purified via flash column chromatography $(0-20 \%$ EtOAc in petroleum ether) to give the titled product as a colorless liquid (31 mg, 57\%): ${ }^{1} \mathrm{H}$ NMR (400 MHz, $\left.\mathrm{CDCl}_{3}\right) \delta 9.71(\mathrm{t}, J=2.3$ $\mathrm{Hz}, 1 \mathrm{H}, H C \mathrm{C}), 7.24-7.20\left(\mathrm{~m}, 3 \mathrm{H}, H_{\mathrm{Ar}}\right), 7.19-7.15\left(\mathrm{~m}, 1 \mathrm{H}, H_{\mathrm{Ar}}\right)$ $3.71\left(\mathrm{~d}, J=2.3 \mathrm{~Hz}, 2 \mathrm{H}, \mathrm{CH}_{2}\right), 2.28\left(\mathrm{~s}, 3 \mathrm{H}, \mathrm{CH}_{3}\right) ;{ }^{13} \mathrm{C}\left\{{ }^{1} \mathrm{H}\right\} \mathrm{NMR}$ $\left(100 \mathrm{MHz}, \mathrm{CDCl}_{3}\right) \delta 199.4(\mathrm{HCO}), 143.3\left(C_{\mathrm{Ar}}\right), 137.3\left(C_{\mathrm{Ar}}\right), 130.8$ $\left(C_{\mathrm{Ar}} \mathrm{H}\right), 130.7\left(C_{\mathrm{Ar}} \mathrm{H}\right), 127.9\left(C_{\mathrm{Ar}} \mathrm{H}\right), 126.6\left(C_{\mathrm{Ar}} \mathrm{H}\right), 48.9\left(\mathrm{CH}_{2}\right), 19.9$ $\left(\mathrm{CH}_{3}\right)$; LRMS (ESI, $\left.m / z\right) 135.3\left([\mathrm{M}+\mathrm{H}]^{+}, 100\right)$; IR $\nu_{\max }$ (film) $2827,1723,1568,1474,1427,1072,782,692 \mathrm{~cm}^{-1}$. The data presented are consistent with literature precedent. ${ }^{48}$

Synthesis of Alcohols from Aryl Hydrazones. General Procedure $E$ for the Synthesis of Alcohols from Aryl Hydrazones. The reaction slug from general procedure $\mathrm{D}$ was extracted with extra EtOAc $(20 \mathrm{~mL} \times 2)$ and washed with water $(20 \mathrm{~mL})$. The combined organic phase was concentrated and redissolved in EtOH $(8 \mathrm{~mL})$. $\mathrm{NaBH}_{4}$ (10 equiv) was added portionwise, and the reaction mixture was stirred for a further $1 \mathrm{~h}$. The resulting mixture was then quenched with ice-water, extracted with ethyl acetae $(2 \times 20 \mathrm{~mL})$, and washed with brine $(2 \times 20 \mathrm{~mL})$. The organic phase was combined, dried over $\mathrm{MgSO}_{4}$, filtered, and concentrated under reduced pressure. The remaining residue was purified via flash column chromatography with appropriate eluents to give the desired alcohol.

2-(4-Chlorophenyl)ethan-1-ol (8a). General procedure E was followed using (4-chlorobenzylidene)hydrazine (0.1 M in EtOAc, 1.0 $\mathrm{mL} / \mathrm{min}$ ), formaldehyde ( $37 \mathrm{wt} \%$ in $\mathrm{H}_{2} \mathrm{O}, 1.0 \mathrm{~mL} / \mathrm{min}$ ), and $\mathrm{NaBH}_{4}$ (152 mg, 10.0 equiv). The crude mixture was purified via flash 
column chromatography (0-20\% EtOAc in petroleum ether) to give the titled product as a colorless liquid (36 mg, 57\%): ${ }^{1} \mathrm{H}$ NMR (600 $\left.\mathrm{MHz} \mathrm{CDCl}_{3}\right) \delta 7.28\left(\mathrm{~d}, J=8.4 \mathrm{~Hz}, 1 \mathrm{H}, H_{\mathrm{Ar}}\right), 7.17(\mathrm{~d}, J=8.4 \mathrm{~Hz}, 1 \mathrm{H}$, $\left.H_{\mathrm{Ar}}\right), 3.85\left(\mathrm{t}, J=6.6 \mathrm{~Hz}, 1 \mathrm{H}, \mathrm{HOCH}_{2}\right), 2.84(\mathrm{t}, J=6.5 \mathrm{~Hz}, 1 \mathrm{H}$, $\left.\mathrm{ArCH}_{2}\right), 1.39$ (br s, $\left.1 \mathrm{H}, \mathrm{HO}\right) ;{ }^{13} \mathrm{C}\left\{{ }^{1} \mathrm{H}\right\}$ NMR $\left(151 \mathrm{MHz}, \mathrm{CDCl}_{3}\right) \delta$ $137.2\left(C_{\mathrm{Ar}}\right), 132.5\left(C_{\mathrm{Ar}}\right), 130.5\left(C_{\mathrm{Ar}} \mathrm{H}\right), 128.8\left(C_{\mathrm{Ar}} \mathrm{H}\right), 63.6$ $\left(\mathrm{HOCH}_{2}\right), 38.6\left(\mathrm{ArCH}_{2}\right)$; LRMS (ESI, $\left.m / z\right) 157.1\left(\left[{ }^{35} \mathrm{M}+\mathrm{H}\right]^{+}\right.$, 100); IR $\nu_{\max }$ (film) 3335, 2932, 1492, 1406, 1090, 1046, 1015, 810 $\mathrm{cm}^{-1}$. The data presented are consistent with literature precedent. ${ }^{49}$

2-(3-Bromophenyl)ethan-1-ol (8b). General procedure E was followed using (3-bromobenzylidene)hydrazine (0.1 M in EtOAc, 1.0 $\mathrm{mL} / \mathrm{min}$ ), formaldehyde ( $37 \mathrm{wt} \%$ in $\mathrm{H}_{2} \mathrm{O}, 1.0 \mathrm{~mL} / \mathrm{min}$ ), and $\mathrm{NaBH}_{4}$ (152 mg, 10.0 equiv). The crude mixture was purified via flash column chromatography (0-20\% EtOAc in petroleum ether) to give the titled product as a colorless liquid (53 mg, 66\%): ${ }^{1} \mathrm{H}$ NMR (400 $\left.\mathrm{MHz}, \mathrm{CDCl}_{3}\right) \delta 7.47-7.29\left(\mathrm{~m}, 2 \mathrm{H}, \mathrm{H}_{\mathrm{Ar}}\right), 7.22-7.06\left(\mathrm{~m}, 2 \mathrm{H}, \mathrm{H}_{\mathrm{Ar}}\right)$, $3.85\left(\mathrm{t}, J=6.5 \mathrm{~Hz}, 2 \mathrm{H}, \mathrm{HOCH}_{2}\right), 2.83\left(\mathrm{t}, J=6.5 \mathrm{~Hz}, 2 \mathrm{H}, \mathrm{ArCH}_{2}\right)$, $1.56(\mathrm{~s}, 1 \mathrm{H}) ;{ }^{13} \mathrm{C}\left\{{ }^{1} \mathrm{H}\right\} \mathrm{NMR}\left(100 \mathrm{MHz}, \mathrm{CDCl}_{3}\right) \delta 141.1\left(C_{\mathrm{Ar}}\right), 132.2$ $\left(C_{\mathrm{Ar}} \mathrm{H}\right), 130.2\left(C_{\mathrm{Ar}} \mathrm{H}\right), 129.7\left(C_{\mathrm{Ar}} \mathrm{H}\right), 127.8\left(C_{\mathrm{Ar}} \mathrm{H}\right), 122.7\left(C_{\mathrm{Ar}}\right), 63.4$ $\left(\mathrm{HOCH}_{2}\right)$, $38.9\left(\mathrm{ArCH}_{2}\right)$; LRMS (ESI, $\left.m / z\right) 201.0\left(\left[{ }^{79} \mathrm{M}+\mathrm{H}\right]^{+}\right.$, 100); IR $\nu_{\max }$ (film) 3333, 2945, 1595, 1567, 1473, 1425, 1200, 1071, $1044,997,853,806,777,692,570 \mathrm{~cm}^{-1}$. The data presented are consistent with literature precedent. ${ }^{50}$

2-(o-Tolyl)ethan-1-ol (8c). General procedure E was followed using (2-methylbenzylidene)hydrazine (0.1 M in EtOAc, $1.0 \mathrm{~mL} /$ min), formaldehyde (37 wt \% in $\mathrm{H}_{2} \mathrm{O}, 1.0 \mathrm{~mL} / \mathrm{min}$ ), and $\mathrm{NaBH}_{4}$ (152 mg, 10.0 equiv). The crude mixture was purified via flash column chromatography $(0-20 \%$ EtOAc in petroleum ether) to give the titled product as a colorless liquid $(36 \mathrm{mg}, 66 \%):{ }^{1} \mathrm{H}$ NMR (400 $\left.\mathrm{MHz}, \mathrm{CDCl}_{3}\right) \delta 7.21-7.02\left(\mathrm{~m}, 4 \mathrm{H}, \mathrm{H}_{\mathrm{Ar}}\right), 3.84(\mathrm{t}, J=6.9 \mathrm{~Hz}, 2 \mathrm{H}$, $\left.\mathrm{HOCH}_{2}\right), 2.90\left(\mathrm{t}, J=6.9 \mathrm{~Hz}, 2 \mathrm{H}, \mathrm{ArCH}_{2}\right), 2.35\left(\mathrm{~s}, 3 \mathrm{H}, \mathrm{CH}_{3}\right)$; ${ }^{13} \mathrm{C}\left\{{ }^{1} \mathrm{H}\right\} \operatorname{NMR}\left(100 \mathrm{MHz}, \mathrm{CDCl}_{3}\right) \delta 136.7\left(C_{\mathrm{Ar}}\right), 136.6\left(C_{\mathrm{Ar}}\right), 130.6$ $\left(C_{\mathrm{Ar}} \mathrm{H}\right), 129.8\left(C_{\mathrm{Ar}} \mathrm{H}\right), 126.7\left(C_{\mathrm{Ar}} \mathrm{H}\right), 126.2\left(C_{\mathrm{Ar}} \mathrm{H}\right), 62.8\left(\mathrm{HOCH}_{2}\right)$, $36.5\left(\mathrm{ArCH}_{2}\right), 19.6\left(\mathrm{CH}_{3}\right)$; LRMS (ESI, $\left.m / z\right) 137.3\left([\mathrm{M}+\mathrm{H}]^{+}\right.$, 100); IR $\nu_{\max }$ (film) 3328, 3017, 2944, 2874, 1604, 1492, 1455, 1379, $1167,1112,1040,938,853,741,612 \mathrm{~cm}^{-1}$. The data presented are consistent with literature precedent. $^{51}$

\section{ASSOCIATED CONTENT}

\section{S Supporting Information}

The Supporting Information is available free of charge on the ACS Publications website at DOI: 10.1021/acs.joc.8b02721.

$$
\text { NMR spectra (PDF) }
$$

\section{AUTHOR INFORMATION}

\section{Corresponding Author}

*E-mail: svl1000@cam.ac.uk.

\section{ORCID $\odot$}

Ricardo Labes: 0000-0003-4001-0775

Patrick Pasau: 0000-0002-1777-7911

Steven V. Ley: 0000-0002-7816-0042

\section{Present Address}

\#(P.D.) School of Chemistry and Chemical Engineering, Queen's University Belfast, David Keir Building, 39-123 Stranmillis Road, Belfast BT9 5AG, U.K.

\section{Author Contributions}

${ }^{\otimes}$ Y.C. and M.L. contributed equally.

\section{Notes}

The authors declare the following competing financial interest(s): D.C.B. is an employee and stockholder of Pfizer, Inc.

${ }^{\perp}$ M.L. was on study leave from the Departmento de Química Orgánica y Farmacéutica, Facultad de Farmacia, Universidad Complutense, 28040 Madrid, Spain.
Additional data related to this publication is available at the University of Cambridge Institutional Data Repository: https://doi.org/10.17863/CAM.27522.

\section{ACKNOWLEDGMENTS}

We thank Vapourtec Ltd. and Duncan Guthrie for the generous loan of a flow UV reactor. Y.C. thanks Pfizer for funding the postdoctoral fellowship. Additional support (to M.L.) from UCB Belgium is also kindly received. The authors also gratefully acknowledge financial support from EPSRC (S.V.L.; Grant Codes EP/K009494/1, EP/K039520/1, and $\mathrm{EP} / \mathrm{M004120/1)}$ and H2020-FETOPEN programme of European commission (S.V.L.; Grant Agreement No. 737266-ONE FLOW).

\section{REFERENCES}

(1) Buchner, E.; Curtius, T. Ueber die Einwirkung von Diazoessigäther auf aromatische Kohlenwasserstoffe. Ber. Dtsch. Chem. Ges. 1885, 18, 2377.

(2) Candeias, N. R.; Paterna, R.; Gois, P. M. P. Homologation Reaction of Ketones with Diazo Compounds. Chem. Rev. 2016, 116, 2937.

(3) Guttenberger, N.; Breinbauer, R. CH and CC bond insertion reactions of diazo compounds into aldehydes. Tetrahedron 2017, 73, 6815.

(4) Mock, W. L.; Hartman, M. E. Synthetic scope of the triethyloxonium ion catalyzed homologation of ketones with diazoacetic esters. J. Org. Chem. 1977, 42, 459.

(5) Allwood, D. M.; Blakemore, D. C.; Ley, S. V. Preparation of Unsymmetrical Ketones from Tosylhydrazones and Aromatic Aldehydes via Formyl C-H Bond Insertion. Org. Lett. 2014, 16, 3064.

(6) Moebius, D. C.; Kingsbury, J. S. Catalytic Homologation of Cycloalkanones with Substituted Diazomethanes. Mild and Efficient Single-Step Access to $\alpha$-Tertiary and $\alpha$-Quaternary Carbonyl Compounds. J. Am. Chem. Soc. 2009, 131, 878.

(7) Hock, K. J.; Koenigs, R. M. The Generation of Diazo Compounds in Continuous-Flow. Chem. - Eur. J. 2018, 24, 10571.

(8) Rulliere, P.; Benoit, G.; Allouche, E. M. D.; Charette, A. B. Safe and Facile Access to Nonstabilized Diazoalkanes Using Continuous Flow Technology. Angew. Chem., Int. Ed. 2018, 57, 5777.

(9) Mastronardi, F.; Gutmann, B.; Kappe, C. O. Continuous Flow Generation and Reactions of Anhydrous Diazomethane Using a Teflon AF-2400 Tube-in-Tube Reactor. Org. Lett. 2013, 15, 5590.

(10) Rossi, E.; Woehl, P.; Maggini, M. Scalable in Situ Diazomethane Generation in Continuous-Flow Reactors. Org. Process Res. Dev. 2012, 16, 1146.

(11) Movsisyan, M.; Delbeke, E. I. P.; Berton, J. K. E. T.; Battilocchio, C.; Ley, S. V.; Stevens, C. V. Taming hazardous chemistry by continuous flow technology. Chem. Soc. Rev. 2016, 45, 4892.

(12) Gutmann, B.; Cantillo, D.; Kappe, C. O. Continuous-Flow Technology-A Tool for the Safe Manufacturing of Active Pharmaceutical Ingredients. Angew. Chem., Int. Ed. 2015, 54, 6688.

(13) Plutschack, M. B.; Pieber, B.; Gilmore, K.; Seeberger, P. H. The Hitchhiker's Guide to Flow Chemistry. Chem. Rev. 2017, 117, 11796.

(14) Müller, S. T. R.; Wirth, T. Diazo Compounds in ContinuousFlow Technology. ChemSusChem 2015, 8, 245.

(15) Deadman, B. J.; Collins, S. G.; Maguire, A. R. Taming Hazardous Chemistry in Flow: The Continuous Processing of Diazo and Diazonium Compounds. Chem. - Eur. J. 2015, 21, 2298.

(16) Kockmann, N.; Thenee, P.; Fleischer-Trebes, C.; Laudadio, G.; Noel, T. Safety assessment in development and operation of modular continuous-flow processes. React. Chem. Eng. 2017, 2, 258.

(17) Pezacki, J. P.; Wagner, B. D.; Lew, C. S. Q.; Warkentin, J.; Lusztyk, J. $\Delta 3-1,3,4-$ Oxadiazolines: Photochemical Precursors to Diazoalkanes and sec-Alkanediazonium Ions in Acidic Solution1. J. Am. Chem. Soc. 1997, 119, 1789. 
(18) Majchrzak, M. W.; Bekhazi, M.; Tse-Sheepy, I.; Warkentin, J. Photolysis of 2-alkoxy-.DELTA.3-1,3,4-oxadiazolines. A new route to diazoalkanes. J. Org. Chem. 1989, 54, 1842.

(19) Greb, A.; Poh, J.-S.; Greed, S.; Battilocchio, C.; Pasau, P.; Blakemore, D. C.; Ley, S. V. A Versatile Route to Unstable Diazo Compounds via Oxadiazolines and their Use in Aryl-Alkyl CrossCoupling Reactions. Angew. Chem., Int. Ed. 2017, 56, 16602.

(20) Dingwall, P.; Greb, A.; Crespin, L. N. S.; Labes, R.; Musio, B.; Poh, J.-S.; Pasau, P.; Blakemore, D. C.; Ley, S. V. C-H functionalisation of aldehydes using light generated, non-stabilised diazo compounds in flow. Chem. Commun. 2018, 54, 11685.

(21) Wommack, A. J.; Kingsbury, J. S. Synthesis of Acyclic Ketones by Catalytic, Bidirectional Homologation of Formaldehyde with Nonstabilized Diazoalkanes. Application of a Chiral Diazomethyl(pyrrolidine) in Total Syntheses of Erythroxylon Alkaloids. J. Org. Chem. 2013, 78, 10573.

(22) Wang, C.; Liu, S.; Xing, D.; Wang, X.; Wu, X.; Hu, W. Efficient synthesis of $\alpha$-aryl serine derivatives via three-component reactions of aryldiazoacetates, anilines and formaldehyde. Tetrahedron 2013, 69, 11203.

(23) Hamilton, J. Y.; Morandi, B.; Carreira, E. M. Homologative Trifluoromethylation of Acetals. Synthesis 2013, 45, 1857.

(24) Gilman, H.; Catlin, W. E. Org. Synth. 1926, 6, 22.

(25) Further experimentation using in-line IR monitoring allowed us to quantify that the formation of the diazo compound was insensitive to both water content and dissolved oxygen.

(26) Zhang, C.; Zhang, L.; Jiao, N. Catalyst free approach to benzimidazoles using air as the oxidant at room temperature. Green Chem. 2012, 14, 3273.

(27) Tran, D. N.; Battilocchio, C.; Lou, S.-B.; Hawkins, J. M.; Ley, S. $\mathrm{V}$. Flow chemistry as a discovery tool to access sp2-sp3 crosscoupling reactions via diazo compounds. Chemical Science 2015, 6, 1120.

(28) Roda, N. M.; Tran, D. N.; Battilocchio, C.; Labes, R.; Ingham, R. J.; Hawkins, J. M.; Ley, S. V. Cyclopropanation using flowgenerated diazo compounds. Org. Biomol. Chem. 2015, 13, 2550.

(29) Shi, Z.-C.; Devasagayaraj, A.; Gu, K.; Jin, H.; Marinelli, B.; Samala, L.; Scott, S.; Stouch, T.; Tunoori, A.; Wang, Y.; Zang, Y.; Zhang, C.; Kimball, S. D.; Main, A. J.; Sun, W.; Yang, Q.; Nouraldeen, A.; Yu, X.-Q.; Buxton, E.; Patel, S.; Nguyen, N.; Swaffield, J.; Powell, D. R.; Wilson, A.; Liu, Q. Modulation of Peripheral Serotonin Levels by Novel Tryptophan Hydroxylase Inhibitors for the Potential Treatment of Functional Gastrointestinal Disorders. J. Med. Chem. 2008, 51, 3684.

(30) Kazem Shiroodi, R.; Dudnik, A. S.; Gevorgyan, V. Stereocontrolled 1,3-Phosphatyloxy and 1,3-Halogen Migration Relay toward Highly Functionalized 1,3-Dienes. J. Am. Chem. Soc. 2012, 134,6928

(31) Cabrera-Pardo, J. R.; Trowbridge, A.; Nappi, M.; Ozaki, K.; Gaunt, M. J. Selective Palladium(II)-Catalyzed Carbonylation of Methylene $\beta-\mathrm{C}-\mathrm{H}$ Bonds in Aliphatic Amines. Angew. Chem., Int. Ed. 2017, 56, 11958.

(32) Boehm, J., Charles; Busch-Petersen, J.; Fu, W.; Jin, Q.; Kerns, J. K.; Li, H.; Lin, G.; Lin, X.; Neipp, C. E. Indole Carboxamides as IKK2 Inhibitors. WO2008118724 (A1), 2008.

(33) Tseng, C.-C.; Noordali, H.; Sani, M.; Madhani, M.; Grant, D. M.; Frenneaux, M. P.; Zanda, M.; Greig, I. R. Development of Fluorinated Analogues of Perhexiline with Improved Pharmacokinetic Properties and Retained Efficacy. J. Med. Chem. 2017, 60, 2780.

(34) Vallone, A.; D’Alessandro, S.; Brogi, S.; Brindisi, M.; Chemi, G.; Alfano, G.; Lamponi, S.; Lee, S. G.; Jez, J. M.; Koolen, K. J. M.; Dechering, K. J.; Saponara, S.; Fusi, F.; Gorelli, B.; Taramelli, D.; Parapini, S.; Caldelari, R.; Campiani, G.; Gemma, S.; Butini, S. Antimalarial agents against both sexual and asexual parasites stages: structure-activity relationships and biological studies of the Malaria Box compound 1-[5-(4-bromo-2-chlorophenyl)furan-2-yl]-N-[(piperidin-4-yl)methyl]methanamine (MMV019918) and analogues. Eur. J. Med. Chem. 2018, 150, 698.
(35) Madder, A.; Sebastian, S.; Van Haver, D.; De Clercq, P. J.; Maskill, H. Mechanism of esterification of 1,3-dimethylamino alcohols by $\mathrm{N}$-acetylimidazole in acetonitrile and the influence of alkyl and geminal dialkyl substitution upon the rate. J. Chem. Soc., Perkin Trans. 2 1997, 2, 2787.

(36) Jones, G. B.; Wright, J. M.; Plourde, G. W.; Hynd, G.; Huber, R. S.; Mathews, J. E. A Direct and Stereocontrolled Route to Conjugated Enediynes. J. Am. Chem. Soc. 2000, 122, 1937.

(37) Callahan, J. F.; Kerns, J. K.; Li, P.; Li, T.; McCleland, B. W.; Nie, H.; Pero, J. E.; Davies, T. G.; Grazia Carr, M.; Griffiths-Jones, C. M.; Heightman, T. D.; Norton, D.; Verdonk, M. L.; Woolford, A. J.A.; Willems, H. M. G. Biaryl Pyrazoles as NRF2 Regulators. WO2017/60854, 2017.

(38) Della, E. W.; Graney, S. D. The Regiochemistry of Cyclization of $\alpha$-Sulfenyl-, $\alpha$-Sulfinyl-, and $\alpha$-Sulfonyl-5-hexenyl Radicals: Procedures Leading to Regioselective Syntheses of Cyclic Sulfones and Sulfoxides. J. Org. Chem. 2004, 69, 3824.

(39) Kitbunnadaj, R.; Hoffmann, M.; Fratantoni, S. A.; Bongers, G.; Bakker, R. A.; Wieland, K.; Jilali, A. e.; De Esch, I. J. P.; Menge, W. M. P. B.; Timmerman, H.; Leurs, R. New high affinity H3 receptor agonists without a basic side chain. Bioorg. Med. Chem. 2005, 13, 6309.

(40) Renou, J.; Dias, J.; Mercey, G.; Verdelet, T.; Rousseau, C.; Gastellier, A.-J.; Arboléas, M.; Touvrey-Loiodice, M.; Baati, R.; Jean, L.; Nachon, F.; Renard, P.-Y. Synthesis and in vitro evaluation of donepezil-based reactivators and analogues for nerve agent-inhibited human acetylcholinesterase. RSC Adv. 2016, 6, 17929.

(41) Kawamoto, T.; Fukuyama, T.; Ryu, I. Radical Addition of Alkyl Halides to Formaldehyde in the Presence of Cyanoborohydride as a Radical Mediator. A New Protocol for Hydroxymethylation Reaction. J. Am. Chem. Soc. 2012, 134, 875.

(42) Jackson, W.; Moffat, M.; Perlmutter, P.; Tasdelen, E. The Stereochemistry of Organometallic Compounds. XXXVIII. Regio- and Stereo-control in the Rhodium-Catalyzed Hydroformylation of Some Alkenyl Phosphites. Aust. J. Chem. 1992, 45, 823.

(43) Nacsa, E. D.; MacMillan, D. W. C. Spin-Center Shift-Enabled Direct Enantioselective $\alpha$-Benzylation of Aldehydes with Alcohols. J. Am. Chem. Soc. 2018, 140, 3322.

(44) Harmata, M.; Gamlath, C. B.; Barnes, C. L.; Jones, D. E. Intramolecular $[4+3]$ Cycloadditions. Studies of Relative Asymmetric Induction. J. Org. Chem. 1995, 60, 5077.

(45) Peram, P. S.; Vences, M.; Schulz, S. A synthetic dodecanolide library for the identification of putative semiochemicals emitted by mantellid frogs. Org. Biomol. Chem. 2017, 15, 6967.

(46) Genovino, J.; Lian, Y.; Zhang, Y.; Hope, T. O.; Juneau, A.; Gagné, Y.; Ingle, G.; Frenette, M. Metal-Free-Visible Light $\mathrm{C}-\mathrm{H}$ Alkylation of Heteroaromatics via Hypervalent Iodine-Promoted Decarboxylation. Org. Lett. 2018, 20, 3229.

(47) Nakaoka, S.; Murakami, Y.; Kataoka, Y.; Ura, Y. Maleimideassisted anti-Markovnikov Wacker-type oxidation of vinylarenes using molecular oxygen as a terminal oxidant. Chem. Commun. 2016, 52, 335

(48) Kavanagh, S. A.; Piccinini, A.; Fleming, E. M.; Connon, S. J. Urea derivatives are highly active catalysts for the base-mediated generation of terminal epoxides from aldehydes and trimethylsulfonium iodide. Org. Biomol. Chem. 2008, 6, 1339.

(49) Cui, X.; Li, Y.; Topf, C.; Junge, K.; Beller, M. Direct Ruthenium-Catalyzed Hydrogenation of Carboxylic Acids to Alcohols. Angew. Chem., Int. Ed. 2015, 54, 10596.

(50) Peng, D.; Zhang, M.; Huang, Z. A General, Practical Triethylborane-Catalyzed Reduction of Carbonyl Functions to Alcohols. Chem. - Eur. J. 2015, 21, 14737.

(51) Toyao, T.; Siddiki, S. M. A. H.; Touchy, A. S.; Onodera, W.; Kon, K.; Morita, Y.; Kamachi, T.; Yoshizawa, K.; Shimizu, K.-i. TiO2Supported $\mathrm{Re}$ as a General and Chemoselective Heterogeneous Catalyst for Hydrogenation of Carboxylic Acids to Alcohols. Chem. Eur. J. 2017, 23, 1001. 